\title{
Genomic Prediction of Strawberry Resistance to Postharvest Fruit Decay Caused by the Fungal Pathogen Botrytis cinerea
}

\author{
Stefan Petrasch*, Saskia D. Mesquida-Pesci*, Dominique D.A. Pincot*, Mitchell J. Feldmann*, Cindy M. López*, Randi Famula*, \\ Michael A. Hardigan*, Glenn S. Cole*, Steven J. Knapp*,1 and Barbara Blanco-Ulate*,1 \\ *Department of Plant Sciences, University of California, Davis, One Shields Avenue, Davis, California, 95616, USA
}

\section{INTRODUCTION}

\begin{abstract}
Gray mold, a disease of strawberry (Fragaria $\times$ ananassa) caused by the ubiquitous necrotroph Botrytis cinerea, renders fruit unmarketable and causes economic losses in the postharvest supply chain. To explore the feasibility of selecting for increased resistance to gray mold, we undertook genetic and genomic prediction studies in strawberry populations segregating for fruit quality and shelf life traits hypothesized to pleiotropically affect susceptibility. As predicted, resistance to gray mold was heritable but quantitative and genetically complex. While every individual was susceptible, the speed of symptom progression and severity differed. Narrow-sense heritability ranged from 0.38-0.71 for lesion diameter (LD) and 0.39-0.44 for speed of emergence of external mycelium (EM). Even though significant additive genetic variation was observed for LD and EM, the phenotypic ranges were comparatively narrow and genome-wide analyses did not identify any large effect loci. Genomic selection accuracy ranged from 0.28-0.59 for LD and 0.37-0.47 for EM. Additive genetic correlations between fruit quality and gray mold resistance traits were consistent with prevailing hypotheses: LD decreased as titratable acidity increased, whereas EM increased as soluble solid content decreased and firmness increased. We concluded that phenotypic and genomic selection could be effective for reducing LD and increasing EM, especially in long shelf life populations, but that a significant fraction of the genetic variation for resistance to gray mold was caused by the pleiotropic effects of fruit quality traits that differ among market and shelf life classes.
\end{abstract}

The fleshy fruits produced by strawberry (Fragaria $\times$ ananassa), tomato (Solanum lycopersicum), and many other horticulturally important plants are susceptible to postharvest decay by gray mold, a devastating disease caused by the necrotrophic fungal pathogen Botrytis cinerea (Jarvis 1962; van Baarlen et al. 2007; Williamson et al. 2007; Dean et al. 2012; Elad et al. 2016; Petrasch et al. 2019a). $B$. cinerea can infect most organs of the plant but is especially destructive on ripe fruit and senescent tissues of dicotyledonous hosts (Jarvis 1962; Dewey and Grant-Downton 2016). Gray mold renders strawberries unmarketable and often causes significant

Manuscript compiled: Sunday $6^{\text {th }}$ June, 2021

${ }^{1}$ Corresponding author: Department of Plant Sciences, University of California, Davis, One Shields Avenue, Davis, California, 95616, USA; bblanco@ucdavis.edu and sjknapp@ucdavis.edu. postharvest losses under conditions favorable for pathogen growth (Barritt 1980; Ries 1995; Dean et al. 2012; Petrasch et al. 2019a). The mechanisms of defense against $B$. cinerea are physiologically and genetically complex and markedly differ from the gene-forgene resistance and programmed cell death mechanisms commonly triggered by biotrophic pathogens (Elad and Evensen 1995; Glazebrook 2005; Lorang 2019; Caseys et al. 2021). As with other necrotrophic pathogens, $B$. cinerea pathogenesis is promoted by fruit ripening and host cell death (Elad and Evensen 1995; Elad et al. 2016; Lorang 2019). Consequently, genetic variation for resistance to gray mold tends to be subtle, limited, and quantitative, which undoubtedly underlies the paucity of studies on breeding for resistance to this pathogen (Finkers et al. 2007a; Williamson et al. 2007; Rowe and Kliebenstein 2008; Lorang 2019; Zhang et al. 2019; Caseys et al. 2021). 
Because natural genetic resistance has been insufficient to prevent postharvest gray mold disease development, pre-harvest fungicides are often applied to suppress pathogen growth and minimize postharvest losses (Legard et al. 1997, 2005; Leroux 2007; Elad et al. 2016; Cosseboom et al. 2019). Controlling B. cinerea with fungicides is difficult because the airborne inoculum is present year round, the host-pathogen interactions are complicated, and the pathogen rapidly evolves resistance to fungicides, particularly after repeated applications of specific chemicals (Jarvis 1962; Leroux 2007; Williamson et al. 2007; Cosseboom et al. 2019; Zhang et al. 2019; Caseys et al. 2021). Moreover, pre-harvest foliar applications of fungicides have not been shown to be effective for reducing postharvest gray mold incidence in strawberry fruit possibly because most fruit infections arise from contaminated flower tissues (van Kan 2006; van Baarlen et al. 2007; Williamson et al. 2007; Veloso and van Kan 2018; Petrasch et al. 2019a).

The development of gray mold resistant cultivars has been challenging in strawberry and other hosts because most genotypes are highly susceptible, strong sources of natural genetic resistance have not been identified, and resistance mechanisms are quantitative (Barritt 1980; van Kan 2006; Finkers et al. 2007a,b, 2008; Williamson et al. 2007; Seijo et al. 2008; Lewers et al. 2012; Petrasch et al. 2019a; Zhang et al. 2019; Caseys et al. 2021). The feasibility of selecting for increased resistance to gray mold has not been deeply explored in strawberry, a species where limited studies have been undertaken to shed light on the genetics of resistance and assess genetic variation for resistance (Barritt 1980; Seijo et al. 2008; Lewers et al. 2012). The problem of breeding for resistance to gray mold has been most extensively studied in tomato, albeit without achieving robust or foolproof solutions (Finkers et al. 2007a,b, 2008). Genetic studies in tomato and Arabidopsis leaves have identified multiple small-effect quantitative trait loci (QTL) that only account for a small fraction of the genetic variation for resistance, seldom translate across genetic backgrounds, and have not solved the problem of breeding for resistance to gray mold (Finkers et al. 2007a,b, 2008; Rowe and Kliebenstein 2008). Although genetic studies of similar depth and breadth have not been undertaken in strawberry, previous studies have not uncovered strong sources of resistance to gray mold (Barritt 1980; Bestfleisch et al. 2015; Seijo et al. 2008; Lewers et al. 2012).

We suspected that selection for increased fruit firmness and other fruit quality traits that extend shelf life pleiotropically increased resistance (decreased susceptibility) to gray mold in strawberry. While hypotheses can be formulated from insights gained from genetic studies in tomato and other hosts (Blanco-Ulate et al. 2016a,b; Petrasch et al. 2019b; Zhang et al. 2019; Caseys et al. 2021), natural genetic resistance appears to be negligible and quantitative and additive genetic correlations between gray mold resistance and fruit quality phenotypes are unknown in strawberry (Jarvis 1962; Rhainds et al. 2002; Chandler et al. 2004; Seijo et al. 2008; González et al. 2009; Lewers et al. 2012; González et al. 2013; Bestfleisch et al. 2015; Petrasch et al. 2019a). The susceptibility of strawberry fruit to B. cinerea increases during ripening (Jarvis 1962), which suggests that susceptibility factors accumulate independent of defense mechanisms during fruit maturation and senescence, as is typical for this necrotroph (Williamson et al. 2007; Zhang et al. 2019; Caseys et al. 2021; Silva et al. 2021). Changes in fruit firmness and other fruit quality traits associated with fruit maturation and ripening in tomato have been shown to increase susceptibility to B. cinerea (Blanco-Ulate et al. 2016a,b; Silva et al. 2021). Although previous studies have been somewhat inconclusive in strawberry, firm-fruited cultivars are predicted to be more resistant to B. cinerea than soft-fruited cultivars (Gooding 1976; Barritt 1980). Moreover, ripening-induced differences in proanthocyanidin and anthocyanin accumulation have been predicted to affect $B$. cinerea resistance in tomato and strawberry (Jersch et al. 1989; Zhang et al. 2013; Bassolino et al. 2013).

To more deeply explore the genetics of resistance to gray mold and assess the feasibility of applying genomic selection for increased resistance to gray mold in strawberry, we developed and studied training populations segregating for fruit quality traits predicted to affect shelf life. Genomic prediction approaches are particularly attractive for postharvest traits that are difficult and costly to phenotype in strawberry but still require sufficient accuracy to complement phenotypic selection and achieve genetic gains (Heffner et al. 2010; Jannink et al. 2010; Lin et al. 2014; VanRaden 2020). The training populations for our studies were developed from crosses between firm-fruited long shelf life (LSL) cultivars and soft-fruited short shelf life (SSL) cultivars. Although the gray mold resistance phenotypes of the parents of these populations were unknown, our hypothesis was that selection for extended shelf life has pleiotropically increased resistance to gray mold in strawberry, primarily because fruit of long shelf life cultivars deteriorate more slowly in postharvest storage than those of short shelf life cultivars. We describe a highly repeatable artificial inoculation protocol for gray mold resistance phenotyping developed for the genomic selection studies described herein. Finally, we discuss the prospects for increasing genetic gains for resistance to gray mold through the application of genomic prediction approaches.

\section{MATERIALS AND METHODS}

\section{Plant Materials and Study Design: Shelf Life Assessment of Mod-} ern Long Shelf Life Cultivars

Shelf life studies were conducted with fruit harvested from five day-neutral cultivars ('UCD Royal Royce', 'UCD Valiant', 'UCD Moxie', 'Cabrillo', and 'Monterey') and three 'summer-plant' ('UCD Finn', 'UCD Mojo', and 'Portola') cultivars (the 'UCD' prefixes are hereafter dropped from the cultivar names) grown on organic and conventional farms using the standard production practices of commercial growers in coastal California. The dayneutral cultivars were grown in 20-plant plots on three commercial farms, one organic and two conventional, in Santa Maria and Prunedale, California in 2017-18 with harvests for postharvest studies on June 22 and 27, July 30, and August 1, 2018, 7/30/2018. The summer-plant cultivars were grown in 20-plant plots on three commercial farms, one organic and two conventional, in Oxnard and Santa Maria, CA in 2018-19 with harvests for postharvest studies on September 27 and 30, November 1 and 18, and December 4, 2019.

To assess shelf life and estimate gray mold incidence, fruit was harvested on two dates at each location and stored in a dark walkin cooler maintained at approximately $4^{\circ} \mathrm{C}$ and $90-95 \%$ relative humidity for 21 days postharvest (dph). Harvest dates were June 22 and 27, July 30, and August 1, 2018 for day-neutral cultivars and September 27 and 30, November 1, 18, and 21, and December 4, 2019 for summer-plant cultivars. Two $0.45 \mathrm{~kg}$ samples of fruit were collected at each harvest and placed in $18.4 \mathrm{~cm} \times 12.1 \mathrm{~cm} \times 6.2 \mathrm{~cm}$ vented plastic clamshells, one of which was stored undisturbed for visual phenotyping and another of which was used for destructive fruit quality trait measurements at three time points for summerplant experiment $(0,7$, and 14 days postharvest; dph) and four time points for the day neutral experiment $(0,7,14$, and $21 \mathrm{dph})$. Soluble solid content (SSC $={ }^{\circ}$ Brix), fruit firmness (g-force), fruit weight ( $\mathrm{g} /$ fruit), fungal decay incidence (\% of clamshell), and mar- 
ketability were recorded at each time point. The latter was visually scored on a 1 to 5 scale, where $1=$ very good, $2=$ good, $3=$ fair, $4=$ poor, and 5 = very poor (Mitcham et al. 1996; do Nascimento Nunes 2015). Four fruit were randomly selected from each clamshell at each time point for fruit firmness and SSC measurements. Firmness was measured using a handheld penetrometer (QA Supplies Model FT02) with a $3 \mathrm{~mm}$ probe. Soluble solid content (SSC; ${ }^{\circ}$ Brix) was measured in the juice of macerated fruit with a digital handheld refractometer (Atago Model PAL-1). Statistical analyses of these experiments were separately performed using the $\mathrm{R}$ package Ime4 (Bates et al. 2015) with cultivar as a fixed effect and location, cultivar $\times$ location, harvest, cultivar $\times$ harvest, harvest $\times$ location, and cultivar $\times$ location $\times$ harvest as random effects. Estimated marginal means (EMMs) and linear contrasts between cultivar EMMs were estimated using the R package emmeans (Lenth 2021).

\section{Plant Materials and Study Design: Genetics of Gray Mold Resis- tance}

Seeds of five $F . \times$ ananassa full-sib families were harvested from crosses produced in a greenhouse at UC Davis in the winter of 2018: Royal Royce $\times$ Primella (PI551422), Royal Royce $\times$ Madame Moutot (PI551632), Royal Royce $\times$ Tangi (PI551481), Royal Royce $\times$ Earlimiss (PI551862), and 05C197P002 × 16C108P065. These families constituted the multi-family training population developed for genomic prediction and other analyses. Seeds were scarified and germinated the week of 18-22 June 2018. Seedlings were established and grown in a greenhouse until they were transplanted to the field on 5 October 2018 at the UC Davis Wolfskill Experiment Orchard (WEO), Winters, CA. The field site was prepared with a disk-harrow and ring-roller and smoothed with a spring-tooth harrow. The soil was fumigated on 14 May 2018 with Pic-Clor $60 ®$ (1, 3-dichloropropene 39\% and chloropicrin 59.6\%; Cardinal Professional Products, Woodland, CA) at a rate of $474.7 \mathrm{~kg} / \mathrm{ha}$. Subsequent to fumigation, planting beds were mechanically shaped to a height of $30.5 \mathrm{~cm}$, width of $50.8 \mathrm{~cm}$, base of $121.9 \mathrm{~cm}$ with center-to-center spacing of $152.4 \mathrm{~cm}$ between beds, and a furrow width of $30.5 \mathrm{~cm}$. Seedlings were transplanted to planting beds on 15 October 2018 in a single centered row with $55.9 \mathrm{~cm}$ between plants within the row. The number of individuals that produced sufficient fruit for the postharvest study of resistance to gray mold were $n=86$ for Royal Royce $\times$ Primella, $n=82$ for Royal Royce $\times$ Madame Moutot, $n=78$ for Royal Royce $\times$ Tangi, $n=92$ for Royal Royce $\times$ Earlimiss, and $n=42$ for 05C197P002 $\times 16$ C108P065. The parents were planted from bare-root plants produced in a commercial high-elevation nursery in Dorris, CA. The population was grown through 30 June 2019, irrigated as needed to prevent water stress, and hand weeded throughout the growing season. Fruit were harvested on from 15 May to 7 June 2019. Additional Royal Royce $\times$ Tangi full-sib individuals $(n=155)$ were grown, handled, and phenotyped in 2019-20 exactly as described above for the 201819 experiment (phenotypic data were collected for 233 Royal Royce $\times$ Tangi individuals over two years). Fruit were harvested 8-29 May 2020.

\section{DNA Isolation and SNP Marker Genotyping}

DNA was extracted from $0.2 \mathrm{~g}$ of dried young leaf tissue with the E-Z 96 Plant DNA Kit (Omega Bio-Tek, Norcross, GA) per the manufacturer's instructions, though the protocol was modified by adding Proteinase $\mathrm{K}$ to the lysis buffer to a final concentration of 0.2 $\mathrm{mg} / \mathrm{ml}$ and extending lysis incubation to $45 \mathrm{~min}$. at $65^{\circ} \mathrm{C}$ in order to increase the quality and yield of the DNA. Single nucleotide polymorphisms (SNPs) were genotyped using the 50K Axiom SNP array (Hardigan et al. 2020), and SNP calls were generated using the Affymetrix Axiom Suite (v1.1.1.66). The raw genotypic data were filtered to identify polymorphic SNP markers with clear and well separated homozygous and heterozygous genotypic classes and eliminate individual SNP markers with minor allele frequencies (MAFs) $<0.05$ and any missing data. This process yielded 11,946 SNPs for the training population $(n=380)$ studied in 2019 and 9,962 SNPs for the Royal Royce $\times$ Tangi full-sib population $(n=$ 233) studied in 2019 and 2020.

\section{Gray Mold Resistance Phenotyping}

We developed a high-throughput protocol for postharvest phenotyping of $B$. cinerea disease progression and symptom development on ripe fruit. Spore suspensions of the B. cinerea strain B05.10 (Büttner et al. 1994; Quidde et al. 1998) were produced from spores grown on potato dextrose agar as described by Petrasch et al. (2019b). Uniformly ripe fruit were harvested at sunrise, avoiding fruit that were under- or over-ripe. The fruit were immediately transferred to cold storage $\left(2.5^{\circ} \mathrm{C}\right)$ and inoculated the day of harvest. Several incubation temperatures $\left(2.5,5.0,10.0\right.$, and $\left.20.0^{\circ} \mathrm{C}\right)$ were tested to identify the optimum temperature for $B$. cinerea growth and development with a minimum of contamination from other postharvest decay pathogens. Fruit were placed on 30-cell plastic egg hatching trays with dimensions of $29 \mathrm{~cm} \times 29 \mathrm{~cm}$ and $4.5 \mathrm{~cm} \times 4.5 \mathrm{~cm}$ cells. The fruit were punctured once near the center with a $3 \mathrm{~mm}$ sterile pipette tip to an approximate depth of 1-2 $\mathrm{mm}$. Ten $\mu \mathrm{l}$ of the B. cinerea conidia suspension (500 conidia $/ \mu \mathrm{l}$ ) was placed on the surface of the puncture. The inoculated fruit were incubated in a growth chamber at $10^{\circ} \mathrm{C}$ and $95 \%$ humidity for 14 days. Disease symptoms were assessed daily after inoculation by manually measuring lesion diameter (LD) and determining the number of days until external mycelium (white or gray hyphae) was evident on the surface of the fruit (EM) near the wound site. Fruit were phenotyped until mycelia covered the entire surface of the fruit. Spoiled fruit with infections outside of the inoculation site or caused by decay organisms other than $B$. cinerea were removed from the experiment. Genome-wide association study (GWAS), QTL mapping, and genomic selection (GS) analyses were applied to LD at 8 days post-inoculation (dpi) and EM.

\section{Fruit Quality Phenotyping}

Fruit quality phenotypes were measured on four fruit harvested from individuals in the multi-family population at harvest. The fruit were photographed with a Sony $\alpha 6000$ camera equipped with an E PZ 16-50 mm F3.5-5.6 OSS lens (SONY, Tokyo, Japan). Photographs were processed with a custom macro in Fiji (Schindelin et al. 2012; Rueden et al. 2017) to obtain RGB color metrics (File S1). RGB colors were subsequently converted into Lab colors using the convertColor() function in R (R Core Team 2021). Fruit firmness (maximum resistance g-force) and fruit diameter ( $\mathrm{mm}$ ) was assessed on whole fruit using a TA.XT plus Texture Analyzer with a TA-53 $3 \mathrm{~mm}$ puncture probe (Stable Micro Systems Ltd., Goldaming, United Kingdoms). Fruit samples were frozen at -20 ${ }^{\circ} \mathrm{C}$ in Whirl-Pak ${ }^{\circledR}$ Homogenizer Blender Filter Bags (Nasco, Fort Atkinson, WI, USA) for quantifying titrable acidity (TA; \%), soluble solid content (SSC; ${ }^{\circ}$ BRIX), and total anthocyanin concentration $(\mathrm{AC} ; \mu g / m l)$. TA percentages were quantified with a Metrohm Robotic Titrosampler System from 1-5 ml of the defrosted homogenized fruit juice (Metrohm AG, Herisau, Switzerland). SSC was measured from approximately $200 \mu \mathrm{l}$ of juice on an RX-5000 $\alpha$-Bev Refractometer (ATAGO Co. Ltd., Tokyo, Japan). Total anthocyanin concentration was measured from a $25 \mu \mathrm{l}$ sample of juice in $200 \mu \mathrm{l}$ 
$1 \% \mathrm{HCl}$ in methanol by reading absorption at a wavelength of 520 nm on a Synergy HTX platereader equipped with Gen5 software (Molecular Devices, San Jose, California, USA). A standard curve $(y=s x+i)$ was calculated for quantifying AC using a dilution series of pelargonidin (Sigma Aldrich, St. Louis, MI, USA) from zero to $300 \mu \mathrm{g} / \mathrm{ml}$ in $50 \mu \mathrm{g} / \mathrm{ml}$ increments, where $y$ were absorption readings for the perlagonin dilution series, $s$ was the slope, $x$ was the concentration of perlagonin in the dilution series, and $i$ was the intercept. AC was estimated by $(A-i) / s$, where $A$ was the absorption reading.

\section{Statistical Analyses: Genetic and QTL Mapping and Genome Wide Association Study}

Genome-wide association study (GWAS) approaches were applied to search for marker-trait associations in the multi-family training population and the Royal Royce $\times$ Tangi population. Estimated marginal means (EMMs) for LD and EM were estimated from subsamples and biological replications using the $R$ package emmeans (Lenth 2021). GWAS analyses were applied using the GWAS() function in the R package rrBLUP (Endelman 2011). The genomic relationship matrix was used to correct for population stratification (Endelman 2011). The genomic inflation factor $(\lambda)$ was 0.60 for LD and 0.71 for EM for analyses of the multi-family population and 1.09 for LD and 1.00 for EM for analyses of the Royal Royce $\times$ Tangi population. The Bonferroni-corrected threshold for statistical significance was $-\log _{10}(0.05 / \mathrm{k})$ where $\mathrm{k}$ is the number of SNPs used in the analysis. The Bonferroni-corrected threshold was $-\log _{10}\left(4.2 \times 10^{6}\right)$ for the multi-family population and $-\log _{10}(5.0 \times$ $10^{6}$ ) for the Royal Royce $\times$ Tangi population.

Parent-specific genetic maps were developed for each full-sib family using a custom PERL script pipeline utilizing the R packages BatchMap and onemap (Schiffthaler et al. 2017; Margarido et al. 2007) (File S4). This pipeline was used to bin co-segregating markers, calculate pairwise recombination fractions, assign markers to linkage groups, and estimate linkage disequilibrium (LD) statistics between groups of markers. Specifically, initial linkage groups (representing chromosomal fragments or sub-linkage groups) were assembled using a LOD threshold of 10.0 and a maximum recombination fraction of 0.08 . These thresholds typically generate more linkage groups that chromosomes. These chromosomal fragments or sub-linkage groups were then merged manually based on inter-group linkage disequilibrium statistics and percent-identity against the physical genome (Edger et al. 2019). The RECORD algorithm (Van Os et al. 2005) was used to estimate marker order and genetic distances across a sliding window of 25 markers with a window overlap of 18 markers.

QTL analyses were applied to parent-specific genetic maps within each full-sib family using the scanone() function and HaleyKnott regression (Haley and Knott 1992) as implemented in the $\mathrm{R}$ package qtl (Broman et al. 2003). The null hypothesis of no significant difference between SNP marker genotypes was tested for each locus using backcross equivalent contrasts; specifically, $\bar{y}_{A a}-\bar{y}_{a a}$ for SNP markers segregating in the female parent and $\bar{y}_{a a}-\bar{y}_{A a}$ for SNP markers segregating in the male parent, where $A a$ is a heterozygote and $a a$ is a homozygote. The null hypothesis of no QTL effect was rejected when the likelihood odds (LOD) ratio for the SNP marker effect exceeded the genome-wide LOD significance threshold empirically estimated by permutation with 1,000 randomly drawn samples (Churchill and Doerge 1994).

We searched the F. $\times$ ananassa 'Camarosa' reference genome (Edger et al. 2019) for QTL-associated candidate genes with putative biotic stress or disease resistance gene function annotations.
Gene Ontology (GO) annotations were filtered to identify candidate genes predicted to be involved in plant-pathogen interactions as described by Silva et al. (2020). Candidate genes were annotated using the KEGG Automated Annotation Server (KAAS) (Moriya et al. 2007) pipeline and filtered pathways as described by Silva et al. (2020). The iTAK pipeline was used to predict presence of transcription factors and protein kinases (Zheng et al. 2016).

\section{Statistical Analyses: Estimation of Genetic and Genomic Predic-} tion Parameters

The repeatability on a progeny mean-basis $(R)$ was estimated for each trait from multiple subsamples/individual (fruit/individual) by $R=\sigma_{B}^{2} /\left(\sigma_{B}^{2}+\sigma_{W}^{2} / \bar{s}\right)$, where $\sigma_{B}^{2}$ is the between-individual variance component, $\sigma_{W}^{2}$ is the among subsamples nested in individuals variance component, $\bar{s}$ is the harmonic mean number of subsamples/individual, and $\bar{s}$ was 2.4 in the multi-family population and 3.6 in the Royal Royce $\times$ Tangi population. Variance components were estimated using REML in the R package lme4 (Bates et al. 2015). Narrow-sense genomic heritability $\left(h^{2}\right)$ was estimated for each trait from the mean of 1,000 REML estimates of the additive genetic and phenotypic variance components from 1,000 Markov Chain Monte Carlo (MCMC) samples drawn for cross-validation using G-BLUP (Endelman 2011).

Genomic estimated breeding values (GEBVs) were estimated for each trait by applying three whole-genome regression methods in both training populations-genomic best linear unbiased prediction (G-BLUP), reproducing kernel Hilbert spaces (RKHS), and support vector machine (SVM). G-BLUP, RKHS, and SVM mixed model analyses were performed using the kinBLUP() function of $\operatorname{rrBLUP}$ (Endelman 2011), the BGLR() function of the $\mathrm{R}$ package BGLR (Pérez and de Los Campos 2014), and the svm() function of R package e1071 (Meyer et al. 2019), respectively. The kernel for RKHS was determined using the multi-kernel averaging method (de Los Campos et al. 2010). Cross-validation analyses were performed for each population $\times$ trait $\times$ whole genome regression (WGR) method using 1,000 MCMC samples/analysis, where GEBVs were estimated from a random sample of $80 \%$ of the individuals and predicted for the GEBVs for the other $20 \%$ of the individuals in each sample: $100 \%$ of the subsamples/individual were used for these analyses. We replicated these cross-validation analyses using a single randomly selected subsample/individual, where GEBVs were estimated from a random sample of $80 \%$ of the individuals (with a single subsample/individual) and predicted the GEBVs for the other $20 \%$ of the individuals. Finally, these cross-validation analyses were repeated by randomly selecting a single subsample/individual from $100 \%$ of the individuals and estimating the correlation between the single-subsample GEBVs with the EMMs estimated from $100 \%$ of the subsamples/individual. Genomic selection accuracy was estimated for each of the nine analyses as the correlation between the phenotypic means (EMMs) and GEBVs $\left(r_{\bar{y}, G E B V}\right)$ for each trait from 1,000 MCMC samples/analysis.

\section{Data Availability}

Genotypic (File S2), phenotypic (File S3), and other supplemental data files, figures, and tables are centrally available at https: //figshare.com). File S1 is a text file with a custom macro developed to process photographic images of strawberry fruit using ImageJ, a "public domain Java image processing program" (https://imagej.nih.gov/ij/index.html). File S2 stores 50K Axiom SNP array genotypic data for the multifamily and the Royal Royce $\times$ Tangi population. File S3 stores the phenotypic data from our study. 
Custom R and PERL scripts developed for genetic mapping analyses are stored in File S4. File S5 stores information on candidate genes linked to gray mold resistance QTL with physical addresses and genome annotations from the $F$. $\times$ ananassa 'Camarosa' v1.0 reference genome (Edger et al. 2019). The latter is available at the Genome Database for Rosaceae (https://www.rosaceae.org/species/ fragaria_x_ananassa/genome_v1.0.a1). File S6 stores the estimated marginal means (EMMs) and genomic-estimated breeding values (GEBVs) for gray mold resistance and fruit quality phenotypes among 380 individuals in a multi-family training population and 233 individuals in the Royal Royce $\times$ Tangi population. Fig. S1 displays time-series photographs of fruit of the long shelf life summer-plant cultivars 'Finn' and 'Mojo' at 0, 7, and 14 days of postharvest storage. Fig. S2 displays the phenotypic distributions and parent EMMs for gray mold resistance phenotypes within full-sib families. Fig. S3 displays kernel densities for genomic prediction accuracy estimated by cross-validation using support vector machine.

\section{RESULTS AND DISCUSSION}

\section{Natural Postharvest Gray Mold Infections on Fruit of Long Shelf Life Cultivars}

Our studies were partly motivated by the observation that gray mold infections were uncommon between 0 and $14 \mathrm{dpi}$ in a series of postharvest shelf life studies of modern long shelf life (LSL) strawberry cultivars (Fig. 1; Fig. S1). The fruit for these studies were harvested from five day-neutral cultivars ('Royal Royce', 'Valiant', 'Moxie', 'Cabrillo', and 'Monterey') and three summerplant cultivars ('Finn', 'Mojo', and 'Portola') grown on commercial farms in coastal California (Fig. 1; Fig. S1). These studies produced several insights. Gray mold infections were rarely observed before 14 dph on any of the cultivars tested (Fig. 1; Fig. S1). Statistically significant differences in gray mold incidence were not observed among day-neutral cultivars ( $p=0.87)$ or summer-plant cultivars $(p=0.98)$. Gray mold incidence ranged from 0.0 to $2.7 \%$ among cultivars at $14 \mathrm{dph}$, a typical postharvest storage duration for LSL cultivars. The five day-neutral cultivars were screened out to 21 $\mathrm{dph}$ to gain insights into the postharvest storage limits for modern LSL cultivars (Fig. 1). Although the fruit were still marketable at $14 \mathrm{dph}$, they became marginally marketable or unmarketable by 17-18 dph (Fig. 1). We observed an exponential increase in gray mold incidence beyond 17-18 dph for every cultivar with means ranging from 10.3 to $36.7 \%$ among day-neutral cultivars at $21 \mathrm{dph}$. These studies showed that gray mold was ubiquitous and eventually rendered the fruit unmarketable but that the natural incidence of gray mold was negligible on fruit of LSL cultivars grown in coastal California within the 14 day postharvest storage window (Fig. 1; File S2).

From previous surveys of phenotypic diversity for resistance to gray mold and common knowledge (Gooding 1976; Barritt 1980; Lewers et al. 2012), we hypothesized that the low incidence of gray mold on commercially produced fruit of LSL cultivars might be genetically correlated with fruit firmness and other fruit quality traits affecting shelf life (Fig. 2). Although phenotypic correlations have been reported (Barritt 1980), genetic correlations have not. The fruit of LSL cultivars are typically much firmer than the fruit of short shelf life (SSL) cultivars commonly grown for local or direct-market consumption, as exemplified by Earlimiss, Madame Moutot, and Primella in the present study (Fig. 2). The latter are sweeter, softer, and perish more rapidly than 'Royal Royce' and other LSL cultivars under normal postharvest storage conditions (Fig. 2). To explore how these phenotypic differences affect resis- tance to gray mold, we developed a training population $(n=380)$ for genomic selection studies by crossing 'Royal Royce', one of the LSL cultivars assessed for natural infections (Fig. 1), with four SSL cultivars ('Earlimiss', 'Madame Moutot', 'Primella', and 'Tangi'), in addition to crossing a pair of LSL parents with differences in fruit firmness and anthocyanin concentration (05C197P002 $\times$ 16C108P065). These full-sib families were phenotyped for resistance to gray mold using an artificial inoculation protocol and genotyped with a 50K Axiom SNP array (Hardigan et al. 2020).

\section{Development of a Highly Repeatable Protocol for Gray Mold Re- sistance Phenotyping in Strawberry}

Natural infections are too inconsistent and unreliable for analyses of the genetics of resistance to gray mold in strawberry. To overcome this problem, we developed a highly repeatable artificial inoculation protocol for gray mold resistance phenotyping that involved puncturing fruit with a $3 \mathrm{~mm}$ probe, propagating spores of a single $B$. cinerea strain (B05.10), introducing a known concentration of spores into the wound site, and monitoring disease development on individual fruit stored undisturbed under high humidity (Fig. 3). Two quantitative B. cinerea disease symptoms were recorded on multiple fruits harvested from training population individuals: water-soaked lesion diameter (LD) in $\mathrm{mm}$ and the number of days post-inoculation (dpi) when external mycelium was observed on the surface of the fruit (EM). We found that incubating artificially inoculated fruit at $10^{\circ} \mathrm{C}$ and $95 \%$ humidity in the dark yielded highly repeatable results with minimal contamination from other postharvest decay pathogens. LD and EM were recorded daily from 1 to $14 \mathrm{dpi}$ (Fig. 3-4). This protocol produced highly reproducible results with repeatability estimates in the 0.660.83 range for LD and 0.68-0.71 range for EM (Table 1). Although critical for maximizing repeatability, this protocol produced more severe disease symptoms than those commonly observed from natural infection, especially on non-wounded fruit of firm-fruited LSL cultivars (Jarvis 1962; Petrasch et al. 2019a) (Fig. 1 and 3; Fig. S1).

\section{Genetics of Resistance to Gray Mold in Strawberry}

To study the genetics of resistance to gray mold in strawberry, artificially inoculated fruit of individuals in multi-family and Royal Royce $\times$ Tangi populations were phenotyped daily for LD and EM over 14 days in cold storage (Fig. 3). The speed of fungal development and symptom severity differed among individuals in both populations (Fig. 4). The phenotypic extremes we observed are illustrated in time-series photographs of four individuals from the upper and lower tails of the LD and EM distributions in the multi-family population (Fig. 3). Lesions became visible and had enlarged to $10.0 \mathrm{~mm}$ by $5 \mathrm{dpi}$ in one of the most susceptible individuals (18C346P030), whereas lesions were not visible until $8 \mathrm{dpi}$ and developed the slowest in one of the least susceptible individuals (18C346P032). Lesions spanned the entire fruit surface of the most susceptible individuals by $8 \mathrm{dpi}$, thereby resulting in significant postharvest fruit deterioration and fungal decay (Fig. 3). Consequently, our genetic analyses of lesion diameter were applied to phenotypes observed $8 \mathrm{dpi}$, the last day in the study that resistance phenotypes could be observed for every individual.

As expected, our analyses confirmed that resistance to gray mold is genetically complex in strawberry, a finding consistent with observations in other hosts (Lurie et al. 1997; Glazebrook 2005; Rowe and Kliebenstein 2008; Lewers et al. 2012; Corwin et al. 2016; Hanson et al. 2016; Petrasch et al. 2019a; Zhang et al. 2019; Silva et al. 2020; Caseys et al. 2021). Although statistically significant dif- 

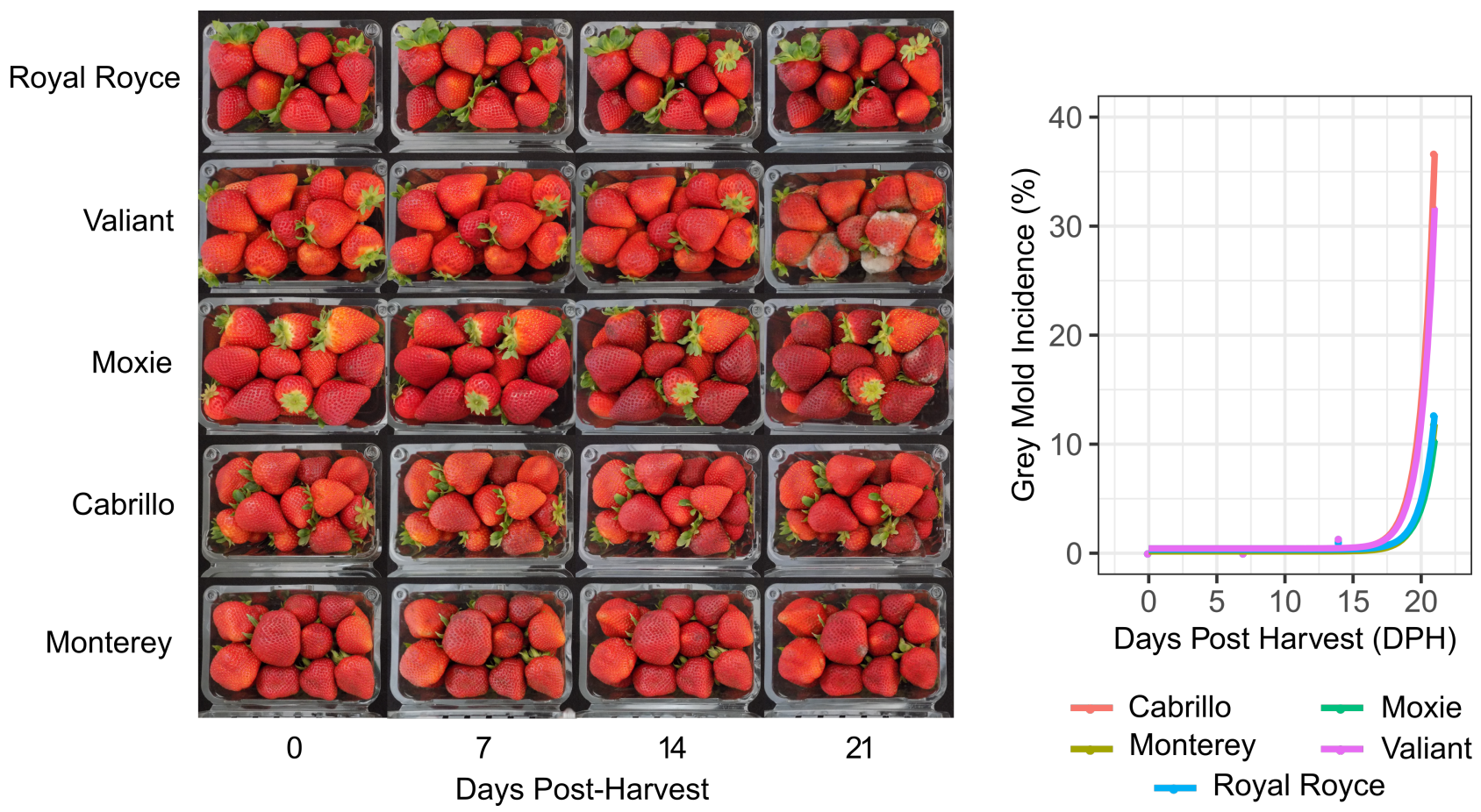

Figure 1 Postharvest Visual Appearance of Cold Stored Fruit of Long Shelf Life Day-Neutral Cultivars Grown in Coastal California. Fruit of 'Royal Royce', 'Valiant', 'Moxie', 'Cabrillo', and 'Monterey' were harvested June 22 and 27, July 30, and August 1, 2018 from commercial farms in Santa Maria and Prunedale, California, immediately cooled, stored undisturbed in $0.45 \mathrm{~kg}$ clamshells at $4^{\circ} \mathrm{C}$ and 90-95\% relative humidity for 21 days postharvest (dph), and photographed and phenotyped 0, 7, 14, and 21 dph (lefthand panel). Estimated marginal means for gray mold incidence (\%) were estimated and plotted and exponential regressions were fit to the original phenotypic observations (right panel). $R^{2}$ estimates for goodness-of-fit of the exponential functions were 0.59 for Cabrillo, 0.22 for Monterey, 0.25 for Moxie, 0.25 for Royal Royce, and 0.50 for Valiant.

$\begin{array}{llccccccc} & \text { 05C197P002 } & \text { 16C108P065 } & \text { Royal Royce } & \text { Primella } & \text { Madame Moutot } & \text { Tangi } & \text { Earlimiss } \\ & & & & & & & & \\ \end{array}$

Figure 2 Fruit Phenotypes for Training Population Parents. Fruit of the parents of Royal Royce $\times$ Primella, Royal Royce $\times$ Madame Moutot, Royal Royce $\times$ Tangi, Royal Royce $\times$ Earlimiss, and 05C197P002 $\times 16$ C108P065 full-sib families. The countries and years of origin are shown for each parent. The fruit firmness categories were soft $\left(<0.15 \mathrm{~kg} / \mathrm{cm}^{2}\right)$, medium $\left(0.15-0.30 \mathrm{~kg} / \mathrm{cm}^{2}\right)$, and firm $(>0.30$ $\left.\mathrm{kg} / \mathrm{cm}^{2}\right)$. The total soluble solids (TSS) categories were low $(<9.0 \%)$, medium $(9.0-11.0 \%)$, and high $(>11.0 \%)$. Titratable acid (TA) concentration $(\%)$ categories were low $(<0.7 \%)$, medium $(0.7-1.0 \%)$, and high $(>1.0 \%)$. External color intensity $(\mathrm{L})$ categories were light ( $\mathrm{L}>41.0)$, medium (25.0-40.0 L), and dark $(<25.0 \mathrm{~L})$. 


\section{Days post inoculation (dpi)}

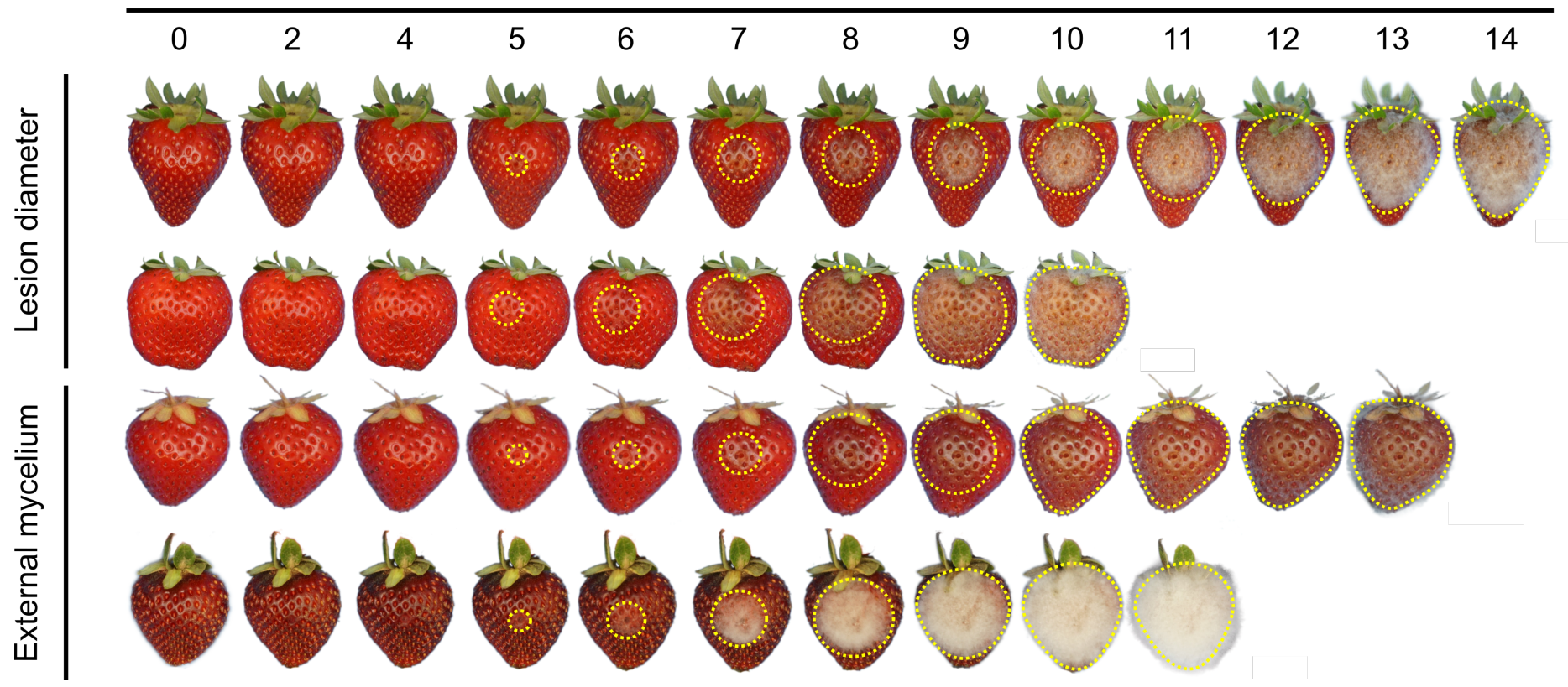

Figure 3 Postharvest Progression of Gray Mold Disease. Fruit were artificially inoculated with the B. cinerea strain B05.10 and phenotyped for 0 to 14 days post-inoculation (dpi). The dotted yellow lines highlight approximate lesion boundaries. The upper panel displays exemplary phenotypic extremes for lesion diameter $(\mathrm{cm})$ among individuals in the multi-family training population. The 18C346P032 individual (first row) had one of the smallest lesion diameters at 8 dpi, whereas the 18C346P030 individual (second row) had one of the largest lesion diameters at $8 \mathrm{dpi}$. The lower panel displays exemplary phenotypic extremes for the speed of appearance of external mycelium on the fruit surface (dpi). The 18C346P025 individual (third row) had one of the slowest, whereas the 18B168P085 individual (fourth row) had one of the fastest times to the appearance of mycelium on the surface of the fruit.

Table 1 Repeatability ( $r$ ) and Narrow-Sense Genomic Heritability $\left(h^{2}\right)$ Estimates for Gray Mold Lesion Diameter (LD) and Speed of Emergence of External Mycelium (EM)

\begin{tabular}{lcccc}
\hline & & & \multicolumn{2}{c}{$\hat{h}^{2}$} \\
Population & Trait & $\hat{r}$ & $\begin{array}{c}\text { Complete } \\
\text { Subsamples }\end{array}$ & $\begin{array}{c}\text { Single } \\
\text { Subsample }\end{array}$ \\
\hline Multi-Family & LD & 0.66 & 0.38 & 0.13 \\
& EM & 0.68 & 0.39 & 0.16 \\
Royal Royce $\times$ Tangi & LD & 0.83 & 0.71 & 0.32 \\
& EM & 0.71 & 0.44 & 0.13 \\
\hline
\end{tabular}

Statistics were estimated for $n=380$ individuals and $s=1,520$ subsamples in a multi-family population and $n=233$ individuals and $s=1,386$ subsamples in the Royal Royce $\times$ Tangi population. The full-sib families in the multi-family population were Royal Royce $\times$ Earlimiss, Royal Royce $\times$ Madame Moutot, Royal Royce $\times$ Primella, Royal Royce $\times$ Tangi, and 05C197P002 $\times 16$ C108P06. Narrow-sense genomic heritability was estimated for $100 \%$ of the subsamples $(\bar{s}=2.9)$ and for a single randomly selected subsample/individual $(\bar{s}=1.0)$ in both populations, where $\bar{s}$ is the harmonic mean number of subsamples/individual.

1 ferences were observed among individuals for LD and EM in both 2 populations $(p<0.01)$, every individual was susceptible and the 3 phenotypic ranges were comparatively narrow (Fig. 3-4). Lesion diameters were approximately normally distributed and ranged from 7.0 to $33.5 \mathrm{~mm}$ at $8 \mathrm{dpi}$ in the multi-family population and 7.0 to $34.0 \mathrm{~mm}$ at $8 \mathrm{dpi}$ in the Royal Royce $\times$ Tangi population (Fig.
4; Fig. S2). Similarly, the speed of appearance of mycelium on the surface of the fruit (EM) was approximately normally distributed and ranged from 4.0 to $12.5 \mathrm{dpi}$ in the multi-family population and 5.5 to $12.5 \mathrm{dpi}$ in the Royal Royce $\times$ Tangi population (fruit were phenotyped out to $14 \mathrm{dpi}$ ). The repeatabilities for LD and EM among individuals in these populations suggested that one-third 

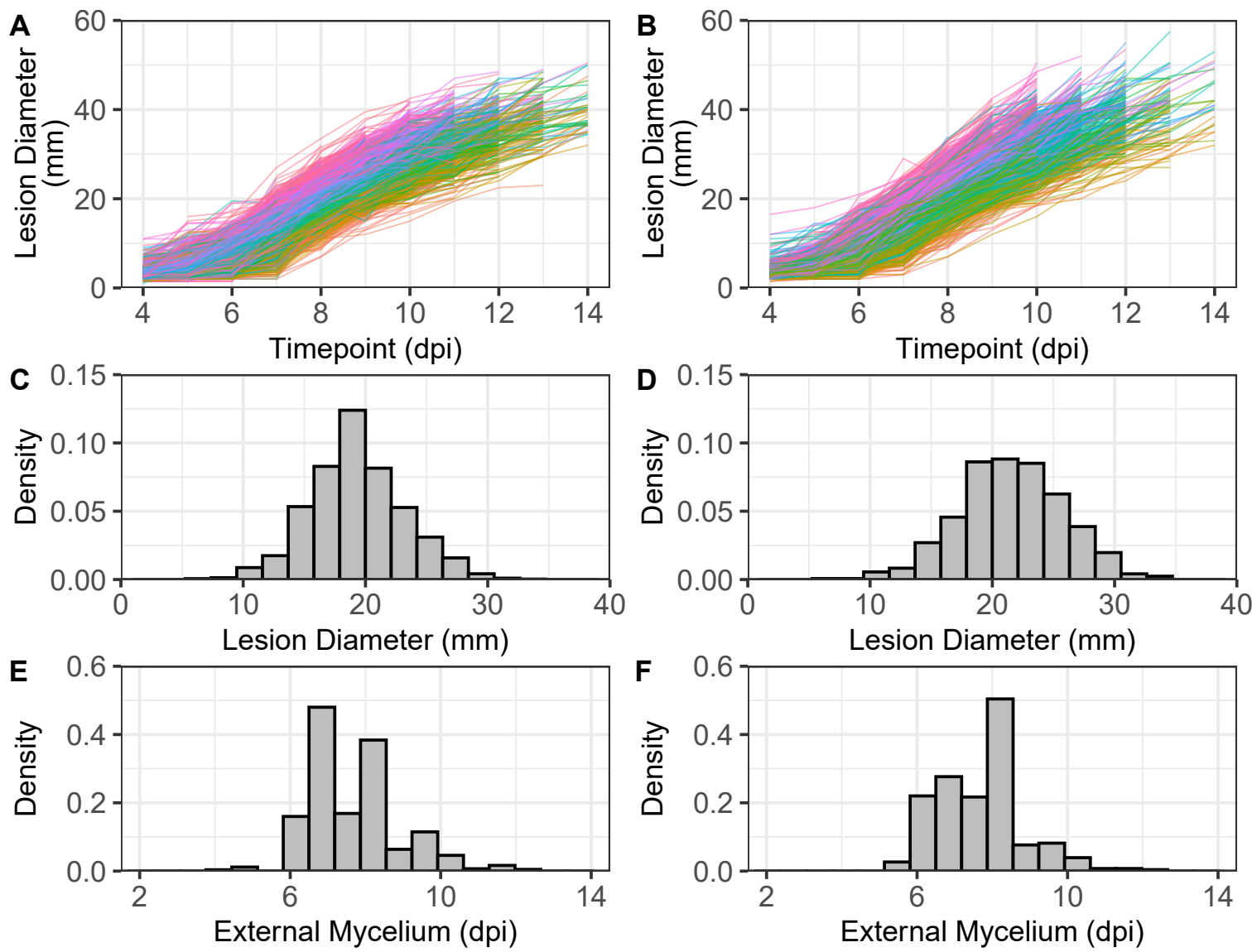

Figure 4 Distributions for Gray Mold Resistance Phenotypes. Fruit of individuals from a multi-family population $(n=380$ individuals and $s=1,520$ fruit) and the Royal Royce $\times$ Tangi population $(n=233$ individuals and $s=1,386$ fruit $)$ were artificially inoculated with $B$. cinerea and phenotyped 0 to 14 days post-inoculation (dpi) for lesion diameter (LD; mm) and the speed of emergence of external mycelium on the surface of the fruit (EM; dpi). The multi-family population was grown in 2018-19, whereas independent samples of Royal Royce times Tangi individuals were grown in 2018-19 and 2019-20. (A) Estimated marginal means (EMM) for 380 individuals in the multi-family population were estimated for each time point from 11,163 observations (1,520 fruit $\times 4$-14 time points) and plotted to visualize changes in LD over time. Because fruit periodically perished and were discarded, the number of observations was less than 21,280 (1,520 fruit $\times 14$ time points). The curves for each individual were plotted with colors corresponding to the EMM rank for lesion diameter at $8 \mathrm{dpi}$. (B) EMMs for 233 individuals in the Royal Royce $\times$ Tangi population were estimated from 9,797 observations (1,386 fruit $\times 4$-14 time points) and plotted and color highlighted as described for the multi-family population. (C and E) Phenotypic distributions for LD and EM in the multi-family population. (D and F) Phenotypic distributions for LD and EM in the Royal Royce $\times$ Tangi population.

or more of the phenotypic variation observed for gray mold resistance was genetically caused (Table 1). Narrow-sense genomic heritability estimates ranged from 0.38 to 0.71 for LD and 0.39 to 0.44 for EM, which suggested that a significant fraction of the genetic variation was additive and thus that resistance to gray mold can be enhanced by artificial selection (Table 1).

Genome-wide searches failed to identify large-effect loci for LD or EM (Fig. 5; Table 2). These searches included GWAS in the training populations and QTL mapping in individual full-sib families. Nine family-specific QTL with small effects were identified, eight for LD and one for EM (Table 2). None had effects large enough to warrant targeting by marker-assisted selection or inclusion as fixed effects in genomic prediction models. Although the QTL effects were small and family-specific (Table 2), a few interesting candidate gene associations were identified when short QTL-associated haploblocks were searched in the reference genome for genes with biotic stress and disease resistance annotations (File S5). A cluster of 11 tandemly duplicated genes encoding pathogenesis-related
(PR) proteins were found in close proximity to the most significant SNP (AX-184469645) associated with a QTL on chromosome 4A (Table 2). These genes share sequence homology to FcPR10, an F. chiloensis ribonuclease encoding gene previously predicted to reduce the severity of gray mold disease in strawberry (González et al. 2009, 2013). The other QTL-associated candidate genes that might warrant further study encode peroxidases (chromosome $7 \mathrm{D} ; \mathrm{Mb} 576-2,709$ ) reported to modulate reactive oxygen species levels and inhibit fungal growth during $B$. cinerea infections (Cantu et al. 2008, 2009; Tomas-Grau et al. 2018) and transcription factors reported to signal pathogen-triggered immunity, e.g., WRKY and AP2/ERF (chromosome 3B; Mb 13,870-15,400) (Gutterson and Reuber 2004; Bigeard et al. 2015), that might target pathogenicity factors, e.g., chitinases (van Schie and Takken 2014) and protease inhibitors (Hermosa et al. 2006; Billon-Grand et al. 2012). While these genes are worthwhile candidates for further study (González et al. 2009, 2013; Petrasch et al. 2019a), the effects of the associated QTL were too small and insignificant for direct selection (Table 
bioRxiv preprint doi: https://doi.org/10.1101/2021.06.08.447540; this version posted June 9, 2021. The copyright holder for this preprint (which was not certified by peer review) is the author/funder, who has granted bioRxiv a license to display the preprint in perpetuity. It is made available under aCC-BY-NC-ND 4.0 International license.
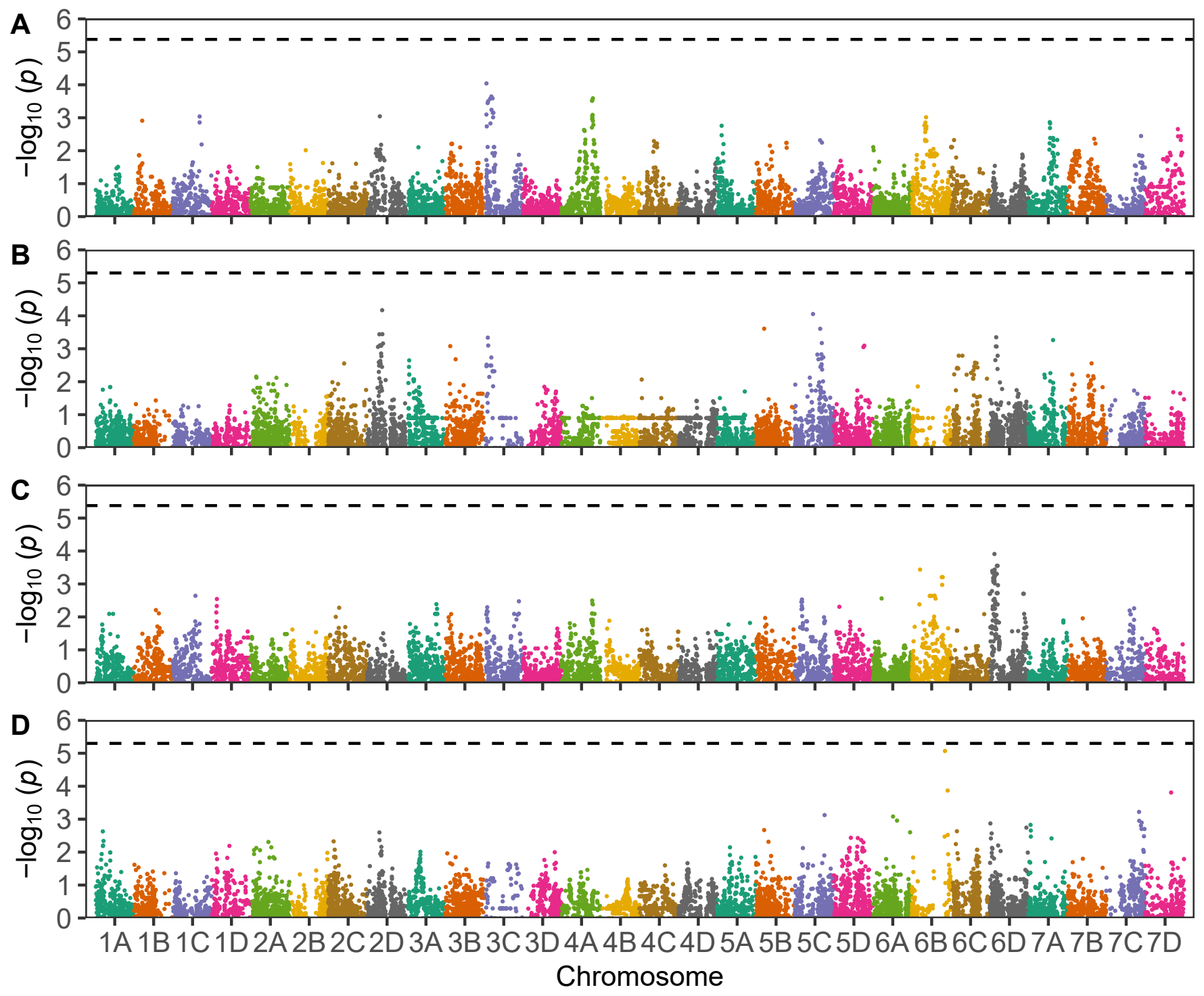

Figure 5 Genome-Wide Association Study of Gray Mold Resistance. Manhattan plots are shown for genome-wide scans for marker-trait associations for lesion diameter (LD) at 8 days post-inoculation (dpi) in the multi-family (A), LD at 8 dpi in the Royal Royce $\times$ Tangi (B), speed of emergence of external mycelium on the surface of the fruit (EM) in the multi-family (C), and EM in the Royal Royce $\times$ Tangi (D) populations. The individuals in both populations were genotyped with a 50K Axiom SNP array. The analyses were done using the 'Camarosa' reference genome (Edger et al. 2019) with physical positions of SNP markers ascertained by (Hardigan et al. 2020) using the chromosome nomenclature of (Hardigan et al. 2021). Horizontal dashed lines identify the genome-wide Bonferroni significance threshold.

2). This was, nevertheless, a first attempt to identify loci underlying resistance to $B$. cinerea in strawberry through a genome-wide search for genotype-to-phenotype associations in the octoploid genome (File S5).

Royal Royce, the firm-fruited LSL parent, was more resistant to gray mold than the soft-fruited SSL parents (phenotypic distributions and parent EMMs for each full-sib family are shown in Fig. S2). Lesions were smaller and mycelium appeared later in Royal Royce than the other parents (resistance increased as LD decreased and EM increased). Royal Royce was the more resistant parent for both traits in the four full-sib families with that parent (Fig. 2; Fig. S2). For the 05C197P002 × 16C108P065 full-sib family, 05C197P002 was more resistant than 16C108P065 for LD and vice versa for EM. The LD and EM differences were highly significant $(p \leq 0.01)$ with individuals transgressing the phenotypic ranges of the parents
(Fig. S2). Transgressive segregation was primarily bidirectional for both traits; however, the EM distributions for Royal Royce $\times$ Tangi and 05C197P002 × 16C108P065 were right-skewed towards more resistance (slower external mycelium emergence) and lacked individuals in the lower tails distal to the more susceptible parent (Fig. S2). These results suggested that favorable alleles were transmitted by both parents for both traits and that favorable alleles for different loci segregated in most of the families.

Lesion diameters were plotted for every individual to visualize phenotypic changes in disease symptoms over time (Fig. 4A-B). The heatmap colors of the individual curves were determined from the lesion diameter EMM ranks at 8 dpi. These plots show that the speed of lesion development differed among individuals, that cross-over individual $\times$ time interactions were negligible, and that the phenotypic changes among individuals were approximately 
bioRxiv preprint doi: https://doi.org/10.1101/2021.06.08.447540; this version posted June 9, 2021. The copyright holder for this preprint (which was not certified by peer review) is the author/funder, who has granted bioRxiv a license to display the preprint in perpetuity. It is made available under aCC-BY-NC-ND 4.0 International license.

Table 2 Summary Statistics for Quantitative Trait Loci Affecting Gray Mold Resistance ${ }^{1}$ in Strawberry

\begin{tabular}{|c|c|c|c|c|c|c|c|}
\hline Population & SNP Marker ${ }^{2}$ & Chromosome $^{3}$ & Position $(\mathrm{Mb})^{4}$ & Contrast $^{5}$ & Effect & $\operatorname{LOD}^{6}$ & Allele $^{7}$ \\
\hline \multicolumn{8}{|c|}{ Lesion Diameter (mm) } \\
\hline 05C197P002 × 16C108P065 & AX-184345814 & $7 \mathrm{D}$ & 0.0 & $A A-A B$ & -3.48 & 4.03 & 05C197P002 \\
\hline Royal Royce $\times$ Primella & AX-184496623 & 3B & 105.0 & BB-AB & 2.49 & 3.61 & Royal Royce \\
\hline Royal Royce $\times$ Primella & AX-184685020 & $5 \mathrm{C}$ & 88.7 & $A A-A B$ & -0.87 & 3.70 & Royal Royce \\
\hline Royal Royce $\times$ Earlimiss & $A X-184026159$ & $3 \mathrm{C}$ & 24.2 & $A A-A B$ & -2.79 & 5.36 & Royal Royce \\
\hline Royal Royce $\times$ Earlimiss & AX-184469645 & $4 \mathrm{~A}$ & 101.6 & $A A-A B$ & 2.52 & 4.26 & Earlimiss \\
\hline Royal Royce $\times$ Tangi & $A X-184718804$ & $3 C$ & 52.8 & BB-AB & 1.92 & 4.21 & Royal Royce \\
\hline Royal Royce $\times$ Tangi & $A X-184031508$ & $5 \mathrm{~A}$ & 0.0 & BB-AB & 1.92 & 4.56 & Royal Royce \\
\hline Royal Royce $\times$ Tangi & AX-184266150 & $7 \mathrm{C}$ & 21.7 & $A A-A B$ & 1.82 & 3.70 & Tangi \\
\hline
\end{tabular}

External Mycelium (dpi)

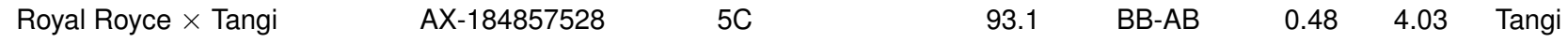

${ }^{1}$ Lesion diameter (LD) and the speed of emergence of external mycelium on the surface of the fruit (EM) were recorded daily from 0 to 14 days post-inoculation (dpi). QTL statistics are shown for LD at $8 \mathrm{dpi}$, the last day that none of the fruit had perished.

${ }^{2}$ Alphanumeric names for SNP markers on the 50K Axiom SNP array Hardigan et al. (2020) with the largest effects (largest LOD score) for a particular QTL.

${ }^{3}$ Chromosome numbers follow the nomenclature proposed by Hardigan et al. (2021) with letters designating subgenomes (A-D) and numbers designating homoeologous chromosomes (1-7).

${ }^{4}$ The physical positions of SNP markers were ascertained by (Hardigan et al. 2020) from alignments of SNP probe sequences to the $F$. $\times$ ananassa 'Camarosa' reference genome (Edger et al. 2019).

${ }^{5}$ SNP marker genotypes were coded $A A, A B$, and $B B$ where $A$ is the allele transmitted by the parent shown on the left in the pedigree and $B$ is the allele transmitted by the parent shown on the right in the pedigree. Contrasts were estimated for each SNP marker as the difference between estimated marginal means (EMMs) for genotypes. The AA-AB contrast compared EMMs between $A A$ homozygotes and $A B$ heterozygotes, whereas the BB-AB contrast compared EMMs between $\mathrm{BB}$ homozygotes and $\mathrm{AB}$ heterozygotes.

${ }^{6}$ Logarithm of the odds (LOD) scores for SNP marker-QTL associations that exceeded statistical thresholds estimated by permutation.

${ }^{7}$ The parent that transmitted the favorable allele. The favorable allele for LD decreased the EMM, whereas the favorable allele for EM increased the EMM.

parallel over time, all of which increased confidence in the heri2 tability of the phenotypic differences we observed (Table 1).

\section{Genomic Prediction Accuracies Varied Between Populations and Symptoms}

Genomic prediction accuracies for different whole-genome regression (WGR) methods ranged from 0.28 to 0.47 for LD and 0.37 to 0.59 for EM when estimated by cross-validation from $100 \%$ of the subsamples (Table 3; Fig. 6-7A, D, G, and J; Fig. S3). The differences in accuracy among WGR methods were mostly negligible (Table 3; Fig. 6-7; Fig. S3). The prediction accuracy was greater for LD than EM in the Royal Royce $\times$ Tangi population (Fig. 6G and J), whereas the reverse was observed in the multi-family population (Fig. 6A and D). Using cross-validation with $100 \%$ of the subsamples, clear differences in prediction accuracy and shrinkage were observed between disease symptoms within and between populations (Table 3; Fig. 6-7A, D, G, and J); Fig. S3). The prediction accuracy for LD was markedly different between the multi-family and Royal Royce $\times$ Tangi populations (Fig. 6A and C). The GEBV range for LD in the multi-family population was half as wide (15.5 to 22.8) and the kernel density was flatter and more vertical than that observed in the Royal Royce $\times$ Tangi population (13.3 to 27.4)
(Fig. 6A and G). Notably, the LD phenotypes of the most resistant individuals in the $\mathrm{RR} \times$ Tangi population (those with the smallest LD means) were well predicted (Fig. 6G). Their EM phenotypes, however, were not as well predicted-the GEBV range for EM (6.8-9.2) was half that of the phenotypic range (5.5-10.7) and the kernel density distribution was flatter and more vertical (Fig. 6-7J; Fig. S3).

One of the challenges of breeding for resistance to gray mold and other postharvest traits is phenotyping throughput. Collectively, 2,563 fruit were harvested and individually stored, tracked, and phenotyped in our study (Fig. 4). Our expectation was that multiple fruit/individual were needed to more accurately estimate EMMs and GEBVs and nominally increase heritability. To assess the effect of subsamples on prediction accuracy and explore the feasibility of applying selection for resistance to gray mold from a single subsample/individual, GEBVs and prediction accuracies were estimated from a single randomly selected subsample/individual. We observed a significant decrease in narrow-sense genomic heritability for LD and EM in the single subsample analyses, e.g., $\hat{h}^{2}$ decreased from 0.38 to 0.13 for LD and 0.39 to 0.16 for EM in the multi-family population (Table 1). Naturally, prediction accuracies plummeted in the single subsample analyses too (Table
22 
bioRxiv preprint doi: https://doi.org/10.1101/2021.06.08.447540; this version posted June 9, 2021. The copyright holder for this preprint (which was not certified by peer review) is the author/funder, who has granted bioRxiv a license to display the preprint in perpetuity. It is made available under aCC-BY-NC-ND 4.0 International license.

Table 3 Genomic Prediction Accuracy for Gray Mold Resistance

\begin{tabular}{|c|c|c|c|c|}
\hline \multirow[b]{2}{*}{ Population ${ }^{1}$} & \multirow[b]{2}{*}{ WGR Method ${ }^{2}$} & \multicolumn{3}{|c|}{ Genomic Prediction Accuracy } \\
\hline & & $\begin{array}{c}\text { Complete Subsample } \\
\text { Cross-Validation }^{3}\end{array}$ & $\begin{array}{l}\text { Single Subsample } \\
\text { Cross-Validation }^{4}\end{array}$ & $\begin{array}{l}\text { Single Subsample } \\
\text { GEBV Prediction }^{5}\end{array}$ \\
\hline \multicolumn{5}{|c|}{ Lesion Diameter (mm) } \\
\hline \multirow[t]{3}{*}{ Multi-Family } & G-BLUP & 0.33 & 0.17 & 0.39 \\
\hline & RKHS & 0.33 & 0.19 & 0.56 \\
\hline & SVM & 0.28 & 0.17 & 0.40 \\
\hline \multirow[t]{3}{*}{ Royal Royce $\times$ Tangi } & GBLUP & 0.52 & 0.56 & 0.61 \\
\hline & RKHS & 0.59 & 0.57 & 0.71 \\
\hline & SVM & 0.59 & 0.58 & 0.63 \\
\hline \multicolumn{5}{|c|}{ External Mycelium (dpi) } \\
\hline \multirow[t]{3}{*}{ Multi-Family } & G-BLUP & 0.47 & 0.35 & 0.44 \\
\hline & RKHS & 0.47 & 0.34 & 0.48 \\
\hline & SVM & 0.44 & 0.40 & 0.49 \\
\hline \multirow[t]{3}{*}{ Royal Royce $\times$ Tangi } & G-BLUP & 0.37 & 0.36 & 0.43 \\
\hline & RKHS & 0.42 & 0.34 & 0.64 \\
\hline & SVM & 0.40 & 0.35 & 0.47 \\
\hline \multicolumn{5}{|c|}{$\begin{array}{l}{ }^{1} \text { Statistics were estimated from analyses of } n=380 \text { individuals and } s=1,520 \text { subsamples in a multi-family pop- } \\
\text { ulation and } n=233 \text { individuals and } s=1,386 \text { subsamples in the Royal Royce } \times \text { Tangi population. The full-sib } \\
\text { families in the multi-family population were Royal Royce } \times \text { Earlimiss, Royal Royce } \times \text { Madame Moutot, Royal Royce } \\
\times \text { Primella, Royal Royce } \times \text { Tangi, and } 05 \mathrm{C} 197 \mathrm{P} 002 \times 16 \mathrm{C} 108 \mathrm{P} 06 \text {. } \\
{ }^{2} \text { Genomic estimated breeding values (GEBVs) were estimated using genomic-best linear unbiased prediction (G- } \\
\text { BLUP), reproducing kernel Hilbert space (RKHS) regression, and support-vector machine (SVM). } \\
{ }^{3} \text { GEBVs and genomic prediction accuracies were estimated from } 100 \% \text { of the subsamples/individual by cross- } \\
\text { validation with } 1,000 \text { permutations using } 80 \% \text { of the individuals for training and } 20 \% \text { of the individuals for prediction, } \\
\text { where the harmonic mean number of subsamples/individual }(\bar{s})=2.9 \text {. } \\
{ }^{4} \text { Genomic prediction accuracy was estimated from a single randomly selected subsample/individual by cross- } \\
\text { validation with } 1,000 \text { permutations using } 80 \% \text { of the individuals for training and } 20 \% \text { of the individuals for prediction. } \\
{ }^{5} \text { Correlation between GEBVs estimated from a single randomly selected subsample/individual and EMMs of individ- } \\
\text { uals estimated from } 100 \% \text { of the subsamples/individual. }\end{array}$} \\
\hline
\end{tabular}

3; Fig. 6-7 and Fig. S3). This is clearly illustrated by the kernel density distributions for genomic selection accuracy estimated for G-BLUP, RKHS, and SVM by cross-validation with a single subsample/individual (Fig. 6-7B, E, H, and K; Fig. S3). GEBV ranges were narrower and kernel density distributions were flatter and more vertical for the single subsample versus multiple subsample analyses for LD and EM in both populations (Fig. 6-7; Fig. S3). Hence, we concluded that breeding values cannot be accurately predicted without subsampling fruit. Nevertheless, a shortcoming of this study was that genomic prediction accuracies could not be compared for equivalent numbers of phenotypic observations for individuals with and without subsamples.

To shed more light on the effect of subsamples, GEBVs were estimated from a single subsample/individual from $100 \%$ of the individuals in each population (Table 3; Fig. 6C, F, I, and L). This analysis was less stringent than either of the cross-validation analy- ses because GEBV estimates were directly compared to EMMs. The prediction accuracies for these analyses were slightly greater than those estimated by 80:20 cross-validation (Table 3; Fig. 6-7 C, F, I, and $\mathrm{L}$ ). These results, while less stringent than the cross-validation analyses, suggested that increasing the number of individuals with fewer subsamples/individual might yield predictions as accurate as those achieved with fewer individuals and an increased number of subsamples/individual, although this needs to be empirically tested and validated.

\section{Gray Mold Resistance Traits Were Genetically Correlated With Shelf Life-Associated Fruit Quality Traits}

One of our original hypotheses was that selection for increased fruit firmness and other shelf life-associated fruit quality traits pleiotropically decreased susceptibility to gray mold in strawberry. The additive genetic correlations observed among the traits pheno- 
bioRxiv preprint doi: https://doi.org/10.1101/2021.06.08.447540; this version posted June 9, 2021. The copyright holder for this preprint (which was not certified by peer review) is the author/funder, who has granted bioRxiv a license to display the preprint in perpetuity. It is made available under aCC-BY-NC-ND 4.0 International license.

A
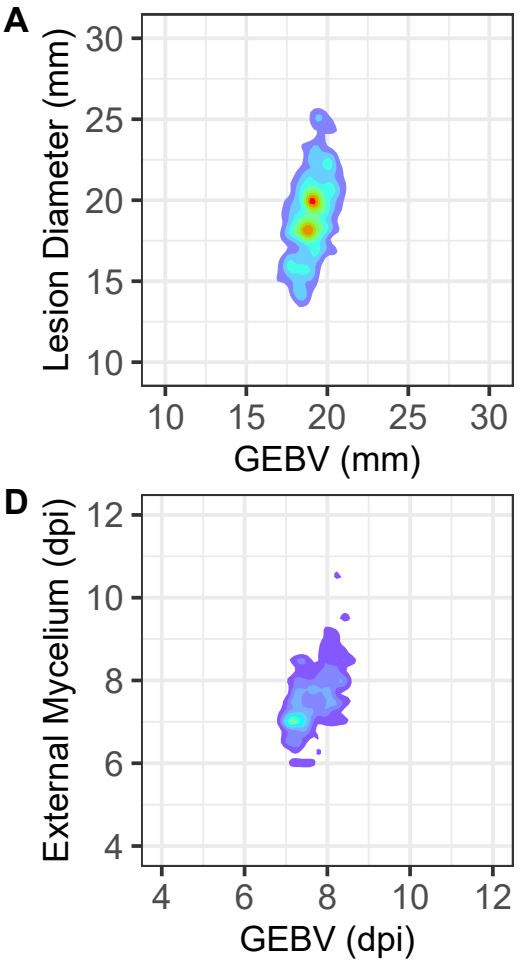

G
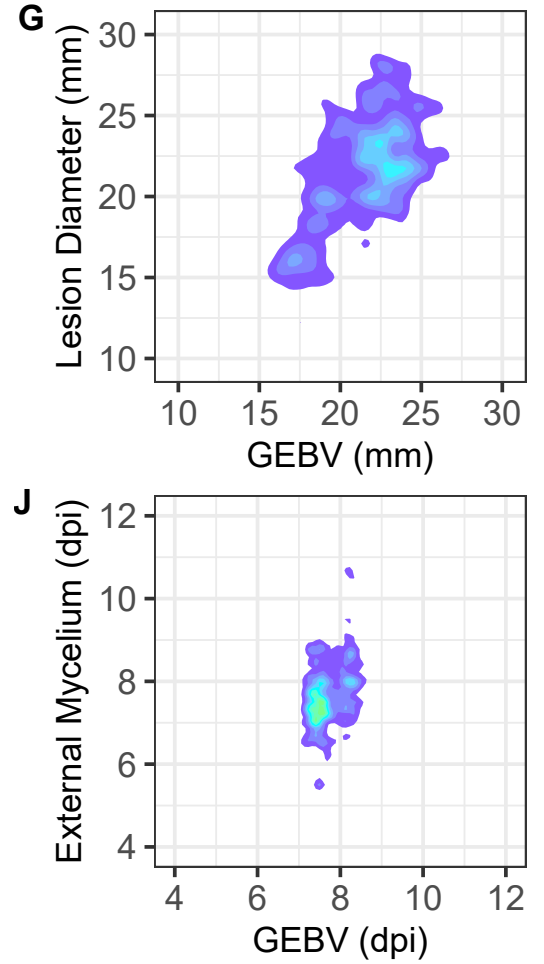

B

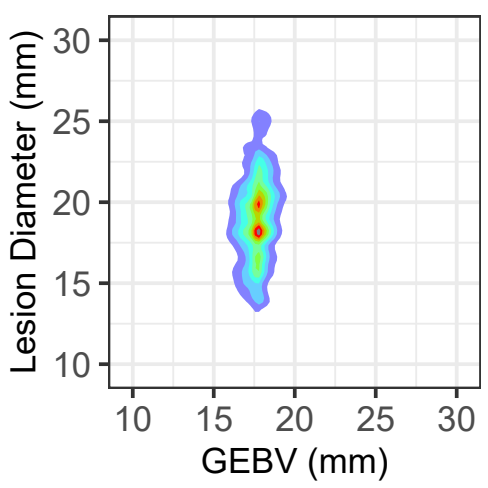

E

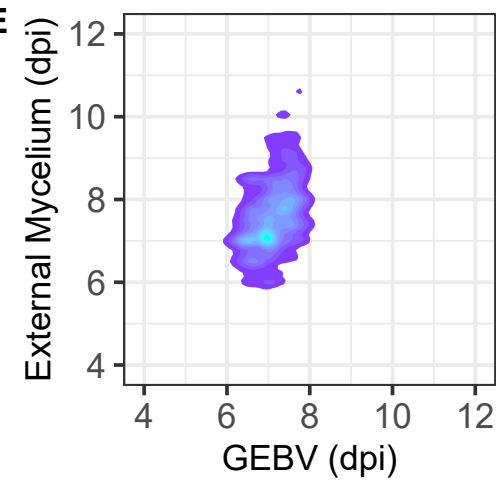

H


C

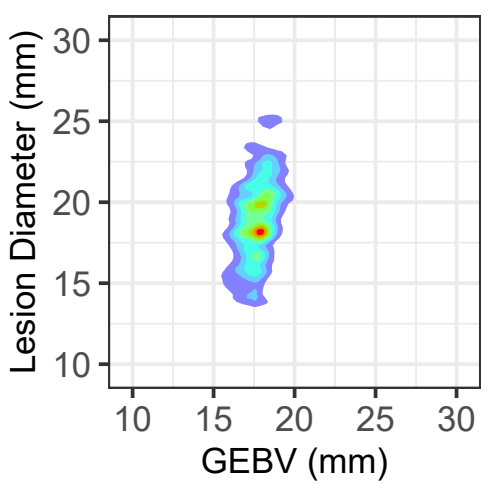

$\mathbf{F}$

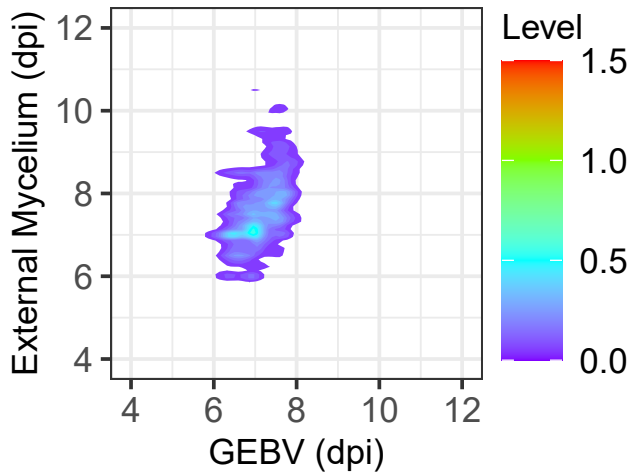

I

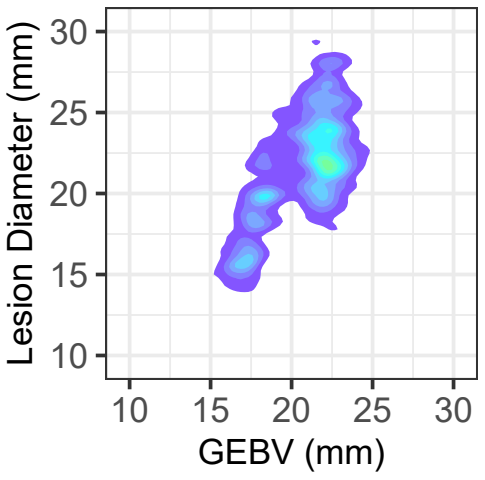

Level

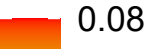

0.06

0.04

0.02

0.00

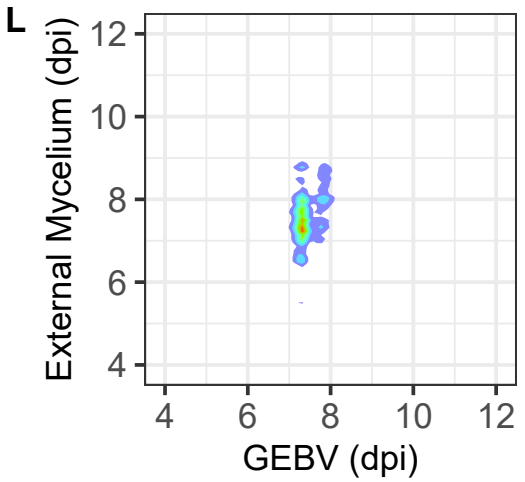

Level

Figure 6 Cross-Validation Accuracy of Genomic Predictions for Gray Mold Resistance. (A-C) Phenotype by genomic estimated breeding value (GEBV) density plots are shown for LD and EM among individuals in a multi-family training population (A-F) and the Royal Royce $\times$ Tangi training population (G-L). GEBVs were estimated using G-BLUP. The density plots shown in the first column (A, D, G, and J) display estimates from 80:20 cross-validation using marginal means (EMMs) estimated from 100\% of the subsamples/individual, where data for $80 \%$ of the individuals were used to estimate GEBVs for the other $20 \%$ of the individuals. There were 380 individuals and a mean of 2.4 subsamples/individual in the multi-family population (1,393 fruit were phenotyped daily) and 233 individuals and a mean of 3.6 subsamples/individual in the Royal Royce $\times$ Tangi population (1,373 fruit were phenotyped daily). The density plots shown in the middle column display estimates from 80:20 cross-validation using phenotypic observations for one randomly chosen subsample/individual, where data for $80 \%$ of the individuals were used to estimate GEBVs for the other $20 \%$ of the individuals. The density plots shown in the last column (C, F, I, and L) display GEBVs estimated from one randomly chosen subsample/individual and EMMs of those individuals estimated from $100 \%$ of the subsamples. 
bioRxiv preprint doi: https://doi.org/10.1101/2021.06.08.447540; this version posted June 9, 2021. The copyright holder for this preprint (which was not certified by peer review) is the author/funder, who has granted bioRxiv a license to display the preprint in perpetuity. It is made available under aCC-BY-NC-ND 4.0 International license.

A

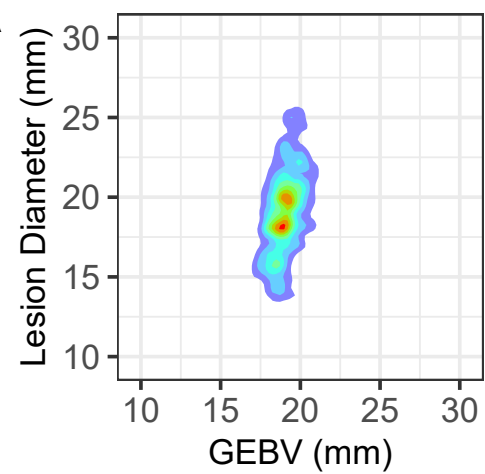

D

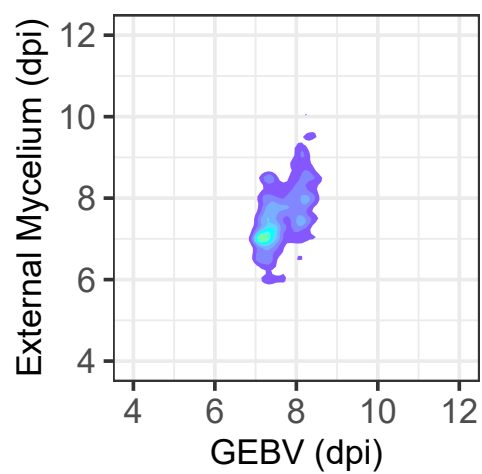

G

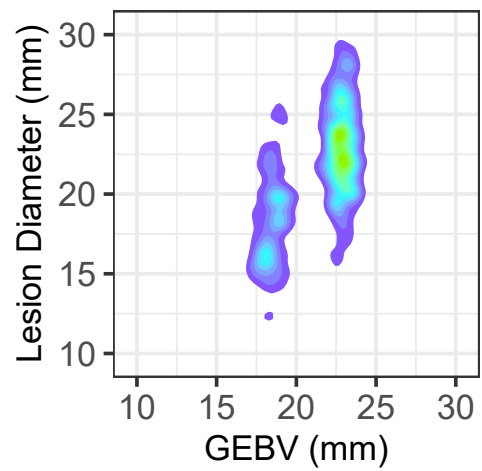

J

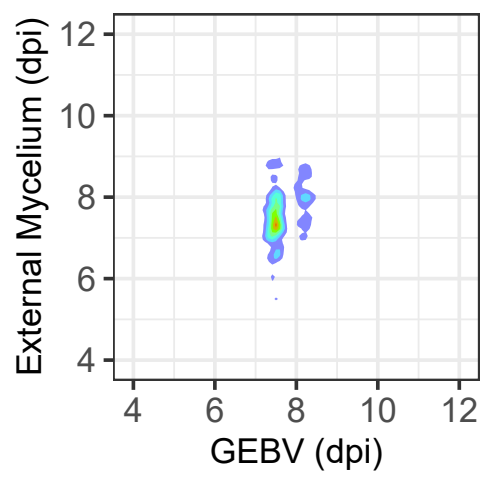

B

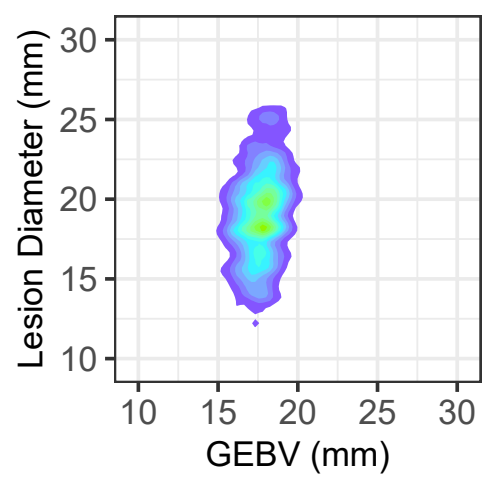

E

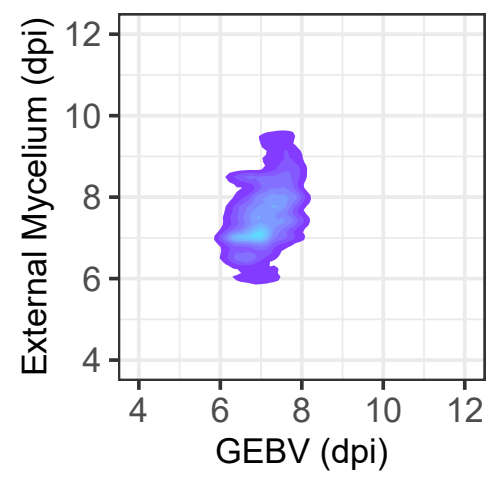

H

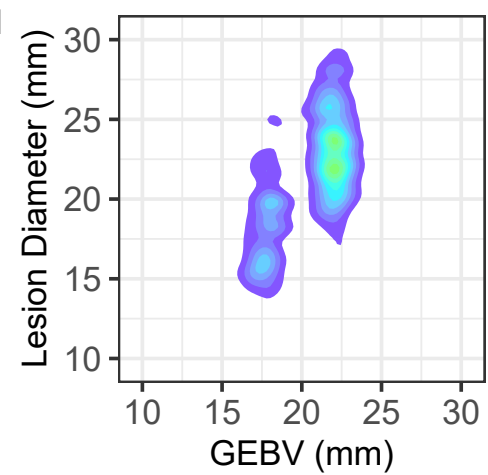

K

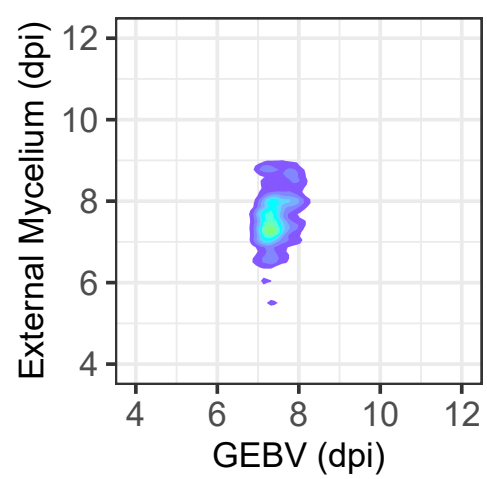

C

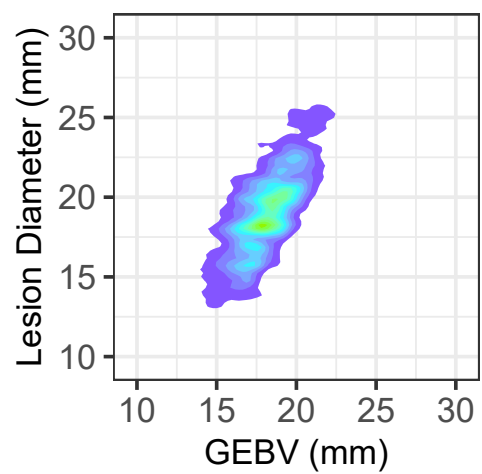

$F$

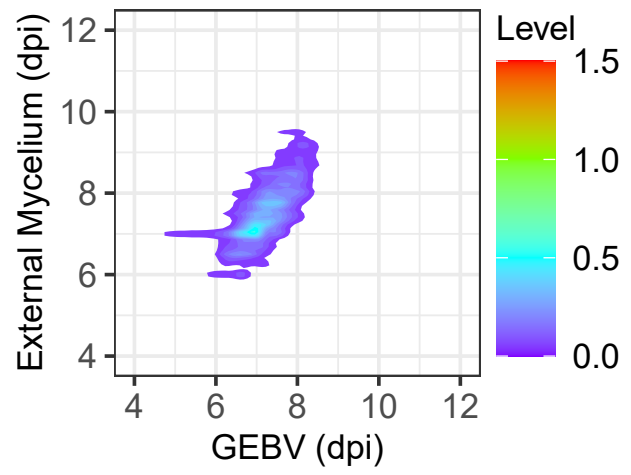

I

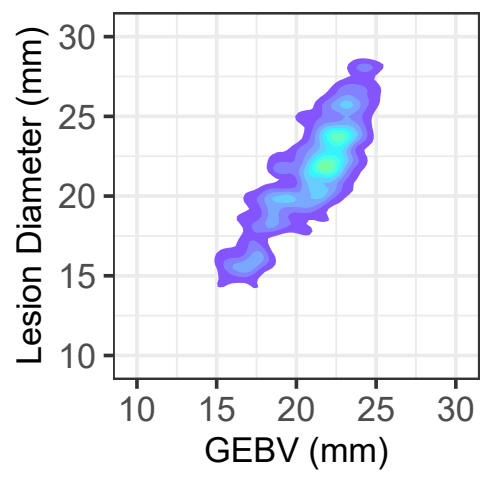

L

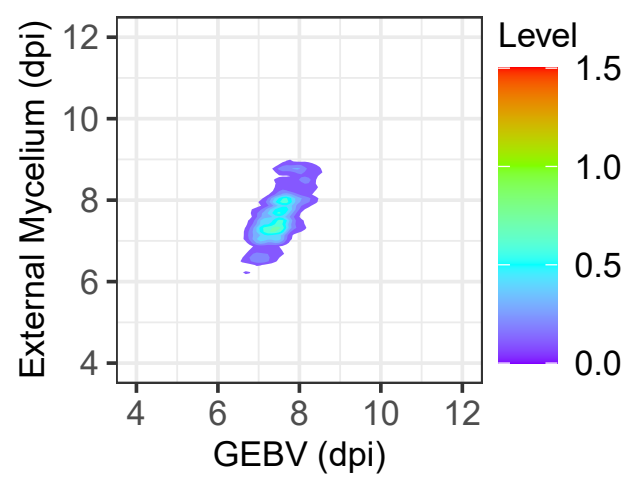

Figure 7 Cross-Validation Accuracy of Genomic Predictions for Gray Mold Resistance. (A-C) Phenotype by genomic estimated breeding value (GEBV) density plots are shown for LD and EM among individuals in a multi-family training population (A-F) and the Royal Royce $\times$ Tangi training population (G-L). GEBVs were estimated using reproducing kernel Hilbert spaces (RKHS) regression. The density plots shown in the first column (A, D, G, and J) display estimates from 80:20 cross-validation using marginal means (EMMs) estimated from $100 \%$ of the subsamples/individual, where data for $80 \%$ of the individuals were used to estimate GEBVs for the other $20 \%$ of the individuals. There were 380 individuals and a mean of 2.4 subsamples/individual in the multi-family population (1,393 fruit were phenotyped daily) and 233 individuals and a mean of 3.6 subsamples/individual in the Royal Royce $\times$ Tangi population (1,373 fruit were phenotyped daily). The density plots shown in the middle column display estimates from 80:20 cross-validation using phenotypic observations for one randomly chosen subsample/individual, where data for $80 \%$ of the individuals were used to estimate GEBVs for the other $20 \%$ of the individuals. The density plots shown in the last column (C, F, I, and L) display GEBVs estimated from one randomly chosen subsample/individual and EMMs of those individuals estimated from $100 \%$ of the subsamples. 

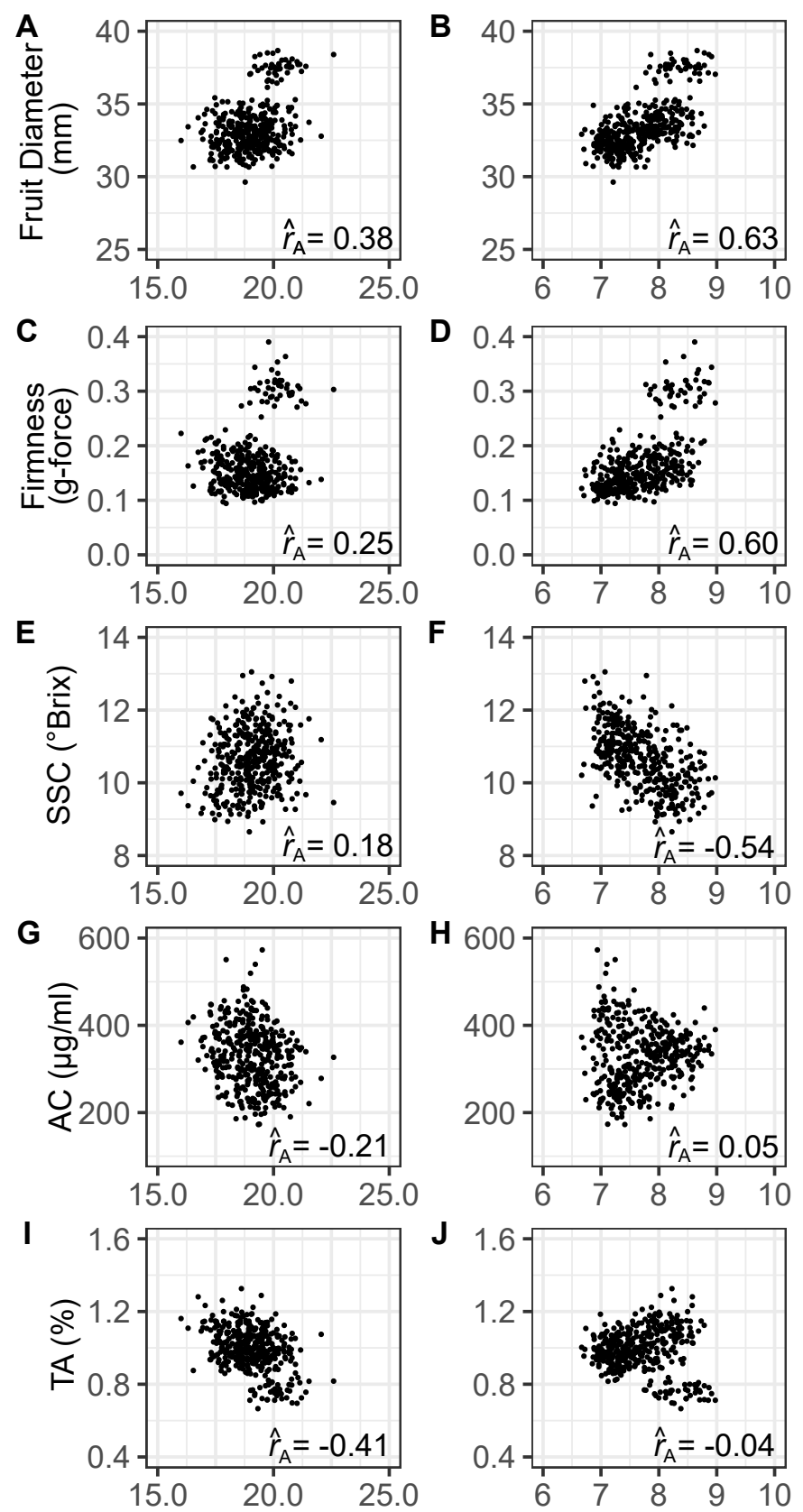

K
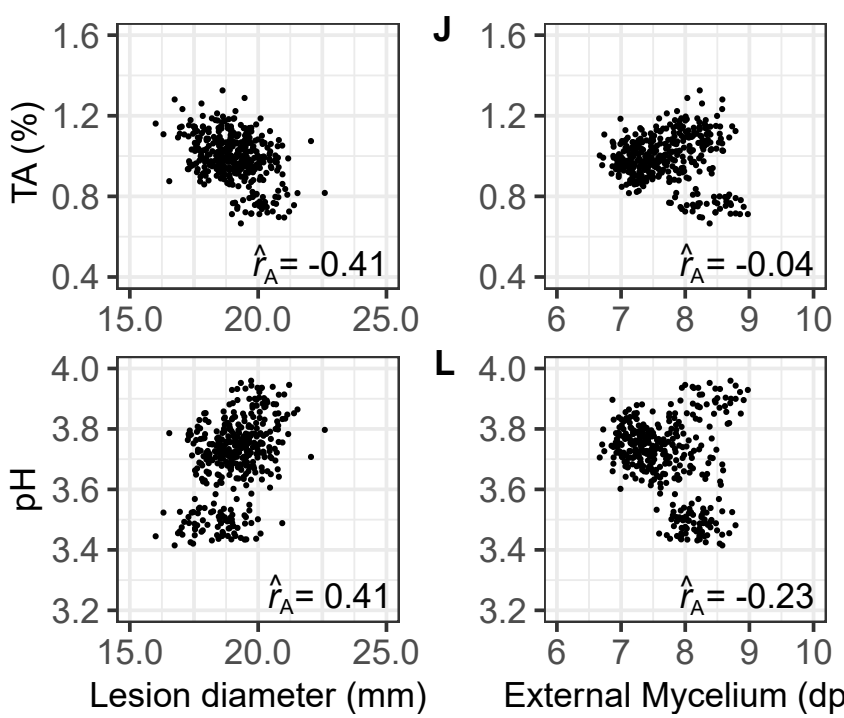

External Mycelium (dpi)

Figure 8 Additive Genetic Correlations Between Gray Mold Resistance and Fruit Quality Traits. Scatter plots are shown for GBLUP estimates of genomic-estimated breeding values (GEBVs) for gray mold resistance and fruit quality traits among 380 individuals in a multi-family training population. Additive genetic correlations $\left(\hat{r}_{A}\right)$ were estimated between lesion diameter (LD), the speed of emergence of external mycelium on the surface of the fruit (EM), fruit diameter, firmness, soluble solids content (SSC), anthocyanin content (AC), titratable acidity (TA), and pH. typed in the present study support this hypothesis (Fig. 8). LD and EM were weakly negatively genetically correlated $\left(\hat{r}_{A}=-0.21\right)$. These gray mold resistance traits were weakly to strongly correlated with fruit quality traits in directions predicted by our hypotheses. Because gray mold resistance increases as LD decreases and EM increases, signs of the additive genetic correlations have different interpretations for LD and EM and can be antagonistic or synergistic. The interpretation depends on the specific phenotypes targeted for a particular market, e.g., SSL versus LSL.

LD was negatively genetic correlated with titratable acidity $\left(\hat{r}_{A}=-0.41\right)$ and positively genetically correlated with $\mathrm{pH}$ $\left(\hat{r}_{A}=0.41\right)$; hence, LD increased as titratable acidity decreased and $\mathrm{pH}$ increased (Fig. 8I-J). The effect of titratable acidity on resistance phenotypes was the motivation for screening additional individuals from the Royal Royce $\times$ Tangi family, which had significant genetic variation for TA and yielded more accurate genomic predictions for LD than were observed in the multi-family population (Fig. 6-7; Fig. S3). EM was more strongly positively genetically correlated with fruit size and firmness than LD and negatively genetically correlated with total soluble solids ( ${ }^{\circ}$ BRIX) 8 . EM increased (disease resistance increased) as BRIX decreased and fruit size and firmness increased. Hence, we found that mycelium developed faster on softer, sweeter, and smaller fruit than firmer, less sweet, and larger fruit, as is typical of Royal Royce and other modern LSL cultivars (Fig. 1-).

Finally, LD was weakly negatively genetically correlated with anthocyanin concentration, whereas EM was uncorrelated with anthocyanin concentration (Fig. 8G-H). Although the additive genetic correlation we observed between LD and anthocyanin concentration was in the direction predicted by previous studies in strawberry and tomato (Jersch et al. 1989; Hébert et al. 2002; Bassolino et al. 2013; Zhang et al. 2013), lesion diameter only slightly decreased as anthocyanin concentration increased (Fig. 8).

\section{Breeding for Enhanced Resistance to Gray Mold in Strawberry}

Breeding for resistance to necrotrophic pathogens has been challenging in plants (Finkers et al. 2007b,a; Williamson et al. 2007; Petrasch et al. 2019a; Delplace et al. 2020; Lorang 2019). The mechanisms of resistance to necrotrophic pathogens are more subtle, quantitative, and complex than those commonly observed for biotrophic pathogens that trigger pathogen-associated molecular pattern-triggered-immunity and effector-triggered immunity (Glazebrook 2005; Jones and Dangl 2006; Jones et al. 2016; Saijo et al. 2018; Zhang et al. 2019; Lorang 2019; Caseys et al. 2021). Our findings were well aligned with previous findings in other B. cinerea hosts and shed light on the genetic complexity of resistance to gray mold in strawberry. Where do we go from here? We are skeptical that significant genetic gains can be achieved for gray mold resistance across the complete shelf life spectrum in strawberry but are confident that postharvest gray mold incidence can be minimized but obviously not eliminated in long shelf life populations. This conclusion seems well aligned with previous findings in strawberry and other hosts of this pathogen (Finkers et al. 2007b,a; Rowe and Kliebenstein 2008; Lewers et al. 2012). Because several fruit quality traits pleiotropically affect gray mold resistance in strawberry, the challenge is exponentially greater when breeding for markets where softer fruits with elevated sugars are preferred and long shelf life phenotypes are neither necessary nor preferred (Fig. 1-2 and 8). However, for markets where LSL cultivars are essential, direct selection for the requisite fruit quality traits seems to confer sufficient resistance to gray mold to ensure marketability under normal postharvest storage conditions and timelines, especially for

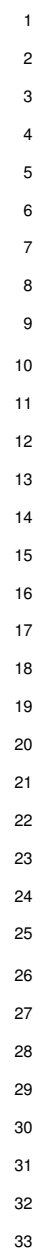

34 35 36 37 38 39 40 41 42 43 44 45 46 47 48 49 50 51 52 53 54 55 56 57 58 59 60 61 
fruit produced in coastal California and other arid and semi-arid environments with low humidity and rainfall (Fig. 1; Fig. S1). Although we only sampled 12 coastal California environments (six locations $\times$ two harvests/location) to estimate the natural incidence of $B$. cinerea among eight LSL cultivars, we suspect that deeper sampling will confirm our findings.

There are open questions to be addressed and were limitations to our study. First, we did not screen diverse germplasm to identify sources of resistance to gray mold. As our study and others have shown, strong sources of resistance to this pathogen may not exist (Chandler et al. 2004; Seijo et al. 2008; Lewers et al. 2012; Bestfleisch et al. 2015). The narrow-sense heritability estimates and genomic prediction results for LD and EM in the present study suggest that a deeper exploration of genetic diversity, while challenging, seems worthwhile (Table 1; Fig. 6-7; Fig. S3). The association between resistance and titratable acidity seems to be particularly promising and worthy of further study (Fig. 8), particularly if increased acidity is offset by increased sugars to achieve a palatable sugar:acid balance.

Second, the parents for this study were selected to assess the effects of fruit quality and shelf life-associated traits on gray mold disease development (Fig. 2), not for known intrinsic differences in gray mold resistance that are genetically uncorrelated with fruit quality and shelf life phenotypes. The pleiotropic effects of shelf life-associated fruit quality traits on gray mold susceptibility appear to be inescapable (Fig. 8). Our results suggest that resistance can be increased by selecting for increased titratable acidity and firmness and decreased sugars but these phenotypes profoundly affect flavor and cannot be manipulated in a vacuum (ZorrillaFontanesi et al. 2011; Diamanti et al. 2012; Lerceteau-Köhler et al. 2012; Verma et al. 2017). Without more extensive germplasm screening, the data needed to guide the selection of parents for future genetic studies are lacking.

Third, the natural incidence of gray mold was only explored in the present study for LSL cultivars commercially grown in California (Fig. 1). Highly perishable short- and medium-shelf life cultivars, as typified by the heirloom cultivars (parents) we screened (Fig. 2), are challenging to grow and phenotype in such studies because they typically have low yields, are easily bruised and wounded, and cannot be harvested and handled with the same robustness as commercially important LSL cultivars. Nevertheless, a study of the natural incidence of gray mold among individuals spanning the shelf life spectrum could shed further light on genetic correlations between fruit quality and gray mold resistance phenotypes and possibly identify sources of favorable alleles underlying intrinsic resistance that are uncorrelated with fruit quality traits, e.g., biochemical phenotypes triggered by defense mechanisms (Diaz et al. 2002; Glazebrook 2005; van Kan 2006; Williamson et al. 2007; Veloso and van Kan 2018; Lorang 2019; Petrasch et al. 2019a). Because gray mold disease symptoms from artificial inoculation protocols are typically harsher than those observed from natural infections of non-wounded fruit (Fig. 1 and 3), a deeper exploration of the natural incidence of gray mold seems warranted in strawberry, perhaps by simulating rainfall in field experiments through overhead irrigation or other practices to increase the uniformity and incidence of natural infection.

Our results suggest that phenotypic or genomic selection could be effective for gray mold resistance but only in certain populations and only when selection for genetically correlated traits does not antagonistically reduce genetic gains for gray mold resistance phenotypes (Table 3; Fig. 6-7; Fig. S3). Genetic gains for gray mold resistance are affected by shelf-life related traits through additive genetic correlations and could be reversed by antagonistically pleiotropically affect gray mold resistance phenotypes (Fig. 8). Genetic variation for fruit quality traits strongly affected the phenotypic differences we observed for LD and EM. Most importantly, the fruit quality traits associated with enhanced flavor were antagonistically genetically correlated with gray mold resistance phenotypes (Fig. 8).

Cross-validation of genomic predictions in the present study shed light on the complexity of genetic mechanisms underlying gray mold resistance phentoypes and highlighted the challenges inherent in breeding for increased resistance to gray mold in strawberry (Table 3; Fig. 6-7; Fig. S3). The three WGR methods we applied to the prediction problem strongly shrunk breeding values to the population mean for LD in the multi-family and EM in the Royal Royce $\times$ Tangi populations. Such shrinkage is typical for moderately heritable complex diseases in plants (Rutkoski et al. 2011, 2014; Poland and Rutkoski 2016).

The prospects for identifying superior genotypes through genomic selection were greater for LD in the Royal Royce $\times$ Tangi population and EM in the multi-family population than vice versa. Whether applying phenotypic or genomic selection, the probability of selecting superior genotypes can be exceedingly low when breeding for resistance to genetically complex diseases in plants (Poland and Rutkoski 2016; Crossa et al. 2017; Voss-Fels et al. 2019). Nevertheless, with cost-effective genome-wide genotyping, genomic selection has the potential to increase genetic gains by increasing the number of selection candidates that can be screened per unit of time and space (Poland and Rutkoski 2016; Crossa et al. 2017). Even in the small populations we studied ( $n=380$ and $n=233$ ), nearly 20,000 phenotypic observations were collected to quantitatively assess resistance to gray mold, which included image analyses at each post-inoculation time point (Fig. 4). The phenotyping throughput needed to effectively evaluate postharvest traits in strawberry can be limiting, particularly when multiple harvests are factored into the equation, e.g., day-neutral cultivars are typically harvested twice weekly over a period of six to eight months in coastal California. Because the progression of disease symptoms was continuous and genotype $\times$ storage time interactions were negligible (Fig. 4), phenotyping throughput can be increased by employing a response surface experiment design, e.g., by phenotyping fruit at three equally spaced time points spanning the range needed to estimate response curves and accurately predict LD and EM phenotypes (Hill and Hunter 1966; Khuri and Mukhopadhyay 2010). This is precisely the sort of breeding problem where genomic prediction methods have the greatest potential utility and applicability (Heffner et al. 2010; Jannink et al. 2010; Lin et al. 2014), despite the complexity of the underlying genetic mechanisms and variable prediction accuracies across populations, which nevertheless affect phenotypic and genomic selection equally (Table 3; Fig. 6-7; Fig. S3).

\section{ACKNOWLEDGEMENTS}

The authors thank Nancy N. Her and Ozalique Williams for assisting with disease assessments and Nayeli Valencia De Puglisi, Bruce Campopiano, and Eduardo Garcia for assisting with fruit harvests.

\section{FUNDING} from the United Stated Department of Agriculture simultaneous selection for fruit quality and shelf life traits that

This research was supported by grants to SJK 
(http://dx.doi.org/10.13039/100000199) National Institute of Food and Agriculture (NIFA) Specialty Crops Research Initiative (\#2017-51181-26833), California Strawberry Commission (http:/ /dx.doi.org/10.13039/100006760), and the University of California, Davis (http://dx.doi.org/10.13039/100007707) and BB-U from UCD College of Agricultural and Environmental Sciences and the Department of Plant Sciences start-up funds. These funding sources supported the dissertation research of SP.

\section{CONFLICT OF INTEREST}

The authors declare no conflict of interest.

\section{LITERATURE CITED}

Barritt, B., 1980 Resistance of strawberry clones to Botrytis fruit rot. J. Am. Soc. Hortic. Sci. 105: 160-164.

Bassolino, L., Y. Zhang, H.-j. Schoonbeek, C. Kiferle, P. Perata, et al., 2013 Accumulation of anthocyanins in tomato skin extends shelf life. New Phytol. 200: 650-655.

Bates, D., M. Mächler, B. Bolker, and S. Walker, 2015 Fitting linear mixed-effects models using lme4. J. Stat. Softw. 67: 1-48.

Bestfleisch, M., M. Luderer-Pflimpfl, M. Höfer, E. Schulte, J. Wünsche, et al., 2015 Evaluation of strawberry (Fragaria L.) genetic resources for resistance to Botrytis cinerea. Plant Pathol. 64: 396405.

Bigeard, J., J. Colcombet, and H. Hirt, 2015 Signaling mechanisms in pattern-triggered immunity (PTI). Mol. Plant 8: 521-539.

Billon-Grand, G., C. Rascle, M. Droux, J. A. Rollins, and N. Poussereau, $2012 \mathrm{pH}$ modulation differs during sunflower cotyledon colonization by the two closely related necrotrophic fungi Botrytis cinerea and Sclerotinia sclerotiorum. Mol. Plant Pathol. 13: 568-578.

Blanco-Ulate, B., J. M. Labavitch, E. Vincenti, A. L. T. Powell, and D. Cantu, 2016a Hitting the wall: Plant cell walls during Botrytis cinerea infections. In Botrytis - the Fungus, the Pathogen and its Management in Agricultural Systems, edited by S. Fillinger and Y. Elad, pp. 361-386, Springer International Publishing, Cham, Switzerland.

Blanco-Ulate, B., E. Vincenti, D. Cantu, and A. L. Powell, 2016b Ripening of tomato fruit and susceptibility to Botrytis cinerea. In Botrytis-the fungus, the pathogen and its management in agricultural systems, pp. 387-412, Springer International Publishing, Cham, Switzerland.

Broman, K. W., H. Wu, Ś. Sen, and G. A. Churchill, 2003 R/qtl: QTL mapping in experimental crosses. Bioinformatics 19: 889-890.

Büttner, P., F. Koch, K. Voigt, T. Quidde, S. Risch, et al., 1994 Variations in ploidy among isolates of Botrytis cinerea: implications for genetic and molecular analyses. Curr. Genet. 25: 445-450.

Cantu, D., B. Blanco-Ulate, L. Yang, J. M. Labavitch, A. B. Bennett, et al., 2009 Ripening-regulated susceptibility of tomato fruit to Botrytis cinerea requires NOR but not RIN or ethylene. Plant Physiol. 150: 1434-1449.

Cantu, D., A. R. Vicente, L. Greve, F. Dewey, A. Bennett, et al., 2008 The intersection between cell wall disassembly, ripening, and fruit susceptibility to Botrytis cinerea. PNAS 105: 859-864.

Caseys, C., G. Shi, N. Soltis, R. Gwinner, J. Corwin, et al., 2021 Quantitative interactions: the disease outcome of Botrytis cinerea across the plant kingdom. G3 jkab175.

Chandler, C. K., J. C. Mertely, and N. Peres, 2004 Resistance of selected strawberry cultivars to anthracnose fruit rot and Botrytis fruit rot. Acta Hortic. 708: 123-126.

Churchill, G. A. and R. W. Doerge, 1994 Empirical threshold values for quantitative trait mapping. Genetics 138: 963-971.
Corwin, J. A., D. Copeland, J. Feusier, A. Subedy, R. Eshbaugh, et al., 2016 The quantitative basis of the arabidopsis innate immune system to endemic pathogens depends on pathogen genetics. PLoS Genet. 12: e1005789.

Cosseboom, S. D., K. L. Ivors, G. Schnabel, P. K. Bryson, and G. J. Holmes, 2019 Within-season shift in fungicide resistance profiles of Botrytis cinerea in california strawberry fields. Plant Dis. 103: 59-64.

Crossa, J., P. Pérez-Rodríguez, J. Cuevas, O. Montesinos-López, D. Jarquín, et al., 2017 Genomic selection in plant breeding: methods, models, and perspectives. Trends Plant Sci. 22: 961-975.

de Los Campos, G., D. Gianola, G. J. Rosa, K. A. Weigel, and J. Crossa, 2010 Semi-parametric genomic-enabled prediction of genetic values using reproducing kernel hilbert spaces methods. Genet. Res. (Camb.) 92: 295-308.

Dean, R., J. A. Van Kan, Z. A. Pretorius, K. E. Hammond-Kosack, A. Di Pietro, et al., 2012 The top 10 fungal pathogens in molecular plant pathology. Mol. Plant Pathol. 13: 414-430.

Delplace, F., C. Huard-Chauveau, U. Dubiella, M. Khafif, E. Alvarez, et al., 2020 Robustness of plant quantitative disease resistance is provided by a decentralized immune network. Proc. Natl. Acad. Sci. 117: 18099-18109.

Dewey, F. M. and R. Grant-Downton, 2016 Botrytis-biology, detection and quantification. In Botrytis - the Fungus, the Pathogen and its Management in Agricultural Systems, edited by S. Fillinger and Y. Elad, pp. 17-34, Springer International Publishing, Cham, Switzerland.

Diamanti, J., F. Capocasa, F. Balducci, M. Battino, J. Hancock, et al., 2012 Increasing strawberry fruit sensorial and nutritional quality using wild and cultivated germplasm. PLoS One 7: e46470.

Diaz, J., A. ten Have, and J. A. van Kan, 2002 The role of ethylene and wound signaling in resistance of tomato to Botrytis cinerea. Plant Physiol. 129: 1341-1351.

do Nascimento Nunes, M. C., 2015 Correlations between subjective quality and physicochemical attributes of fresh fruits and vegetables. Postharvest Biol. Technol. 107: 43-54.

Edger, P. P., T. J. Poorten, R. VanBuren, M. A. Hardigan, M. Colle, et al., 2019 Origin and evolution of the octoploid strawberry genome. Nat. Genet. 51: 541-547.

Elad, Y. and K. Evensen, 1995 Physiological aspects of resistance to Botrytis cinerea. Phytopathology 85: 637-643.

Elad, Y., I. Pertot, A. M. C. Prado, and A. Stewart, 2016 Plant hosts of Botrytis spp. In Botrytis-the fungus, the pathogen and its management in agricultural systems, pp. 413-486, Springer.

Endelman, J. B., 2011 Ridge regression and other kernels for genomic selection with R package rrBLUP. Plant Genome 4: 250255.

Finkers, R., Y. Bai, P. van den Berg, R. van Berloo, F. MeijerDekens, et al., 2008 Quantitative resistance to Botrytis cinerea from Solanum neorickii. Euphytica 159: 83-92.

Finkers, R., P. van den Berg, R. van Berloo, A. Ten Have, A. W. van Heusden, et al., 2007a Three QTLs for Botrytis cinerea resistance in tomato. Theor. Appl. Genet. 114: 585-593.

Finkers, R., A. W. van Heusden, F. Meijer-Dekens, J. A. van Kan, P. Maris, et al., 2007b The construction of a Solanum habrochaites LYC4 introgression line population and the identification of QTLs for resistance to Botrytis cinerea. Theor. Appl. Genet. 114: 1071-1080.

Glazebrook, J., 2005 Contrasting mechanisms of defense against biotrophic and necrotrophic pathogens. Annu. Rev. Phytopathol. 43: 205-227.

González, G., L. Fuentes, M. A. Moya-León, C. Sandoval, and 
R. Herrera, 2013 Characterization of two PR genes from Fragaria chiloensis in response to Botrytis cinerea infection: A comparison with Fragaria $\times$ ananassa. Physiol. Mol. Plant Pathol. 82: 73-80.

González, G., M. Moya, C. Sandoval, and R. Herrera, 2009 Genetic diversity in chilean strawberry (Fragaria chiloensis): differential response to Botrytis cinerea infection. Span. J. Agric. Res. pp. 886-895.

Gooding, H., 1976 Resistance to mechanical injury and assessment of shelf life in fruits of strawberry (Fragaria $\times$ ananassa). Hortic. Res. 16: 71-82.

Gutterson, N. and T. L. Reuber, 2004 Regulation of disease resistance pathways by AP2/ERF transcription factors. Curr. Opin. Plant Biol. 7: 465-471.

Haley, C. S. and S. A. Knott, 1992 A simple regression method for mapping quantitative trait loci in line crosses using flanking markers. Heredity 69: 315-324.

Hanson, P., S.-F. Lu, J.-F. Wang, W. Chen, L. Kenyon, et al., 2016 Conventional and molecular marker-assisted selection and pyramiding of genes for multiple disease resistance in tomato. Sci. Hortic. 201: 346-354.

Hardigan, M. A., M. J. Feldmann, A. Lorant, K. A. Bird, R. Famula, et al., 2020 Genome synteny has been conserved among the octoploid progenitors of cultivated strawberry over millions of years of evolution. Front. Plant Sci. 10: 1789.

Hardigan, M. A., A. Lorant, D. D. Pincot, M. J. Feldmann, R. A Famula, et al., 2021 Unraveling the complex hybrid ancestry and domestication history of cultivated strawberry. Mol. Biol. Evol.

Hébert, C., M. Charles, L. Gauthier, C. Willemot, S. Khanizadeh, et al., 2002 Strawberry proanthocyanidins: biochemical markers for Botrytis cinerea resistance and shelf-life predictability. Acta Hortic. 567: 659-662.

Heffner, E. L., A. J. Lorenz, J.-L. Jannink, and M. E. Sorrells, 2010 Plant breeding with genomic selection: gain per unit time and cost. Crop Sci. 50: 1681-1690.

Hermosa, M., D. Turra, V. Fogliano, E. Monte, and M. Lorito, 2006 Identification and characterization of potato protease inhibitors able to inhibit pathogenicity and growth of Botrytis cinerea. Physiol. Mol. Plant Pathol. 68: 138-148.

Hill, W. J. and W. G. Hunter, 1966 A review of response surface methodology: a literature survey. Technometrics 8: 571-590.

Jannink, J.-L., A. J. Lorenz, and H. Iwata, 2010 Genomic selection in plant breeding: from theory to practice. Brief. Funct. Genom. 9: 166-177.

Jarvis, W., 1962 The infection of strawberry and raspberry fruits by Botrytis cinerea Fr. Ann. Appl. Biol. 50: 569-575.

Jersch, S., C. Scherer, G. Huth, and E. Schlösser, 1989 Proanthocyanidine als Ursache der Quieszenz von Botrytis cinerea in unreifen Erdbeerfrüchten. Z. Pflanzenk. Pflanzen. pp. 365-378.

Jones, J. D. and J. L. Dangl, 2006 The plant immune system. Nature 444: 323-329.

Jones, J. D., R. E. Vance, and J. L. Dangl, 2016 Intracellular innate immune surveillance devices in plants and animals. Science 354: aaf6395-1 - aaf6395-8.

Khuri, A. I. and S. Mukhopadhyay, 2010 Response surface methodology. Wiley Interdiscip. Rev. Comput. Stat. 2: 128-149.

Legard, D., C. Chandler, and J. Bartz, 1997 The control of strawberry diseases by sanitation. Acta Hortic. 439: 917-922.

Legard, D., S. MacKenzie, J. Mertely, C. Chandler, and N. Peres, 2005 Development of a reduced use fungicide program for control of botrytis fruit rot on annual winter strawberry. Plant Dis. 89: 1353-1358.

Lenth, R. V., 2021 emmeans: Estimated Marginal Means, aka Least-
Squares Means. R package version 1.5.5-1.

Lerceteau-Köhler, E., A. Moing, G. Guérin, C. Renaud, A. Petit, et al., 2012 Genetic dissection of fruit quality traits in the octoploid cultivated strawberry highlights the role of homoeo-qtl in their control. Theoretical and Applied Genetics 124: 1059-1077.

Leroux, P., 2007 Chemical control of botrytis and its resistance to chemical fungicides. In Botrytis: Biology, Pathology and Control, pp. 195-222, Springer.

Lewers, K. S., Y. Luo, and B. T. Vinyard, 2012 Evaluating strawberry breeding selections for postharvest fruit decay. Euphytica 186: 539-555.

Lin, Z., B. Hayes, and H. Daetwyler, 2014 Genomic selection in crops, trees and forages: a review. Crop Pasture Sci. 65: 11771191.

Lorang, J., 2019 Necrotrophic exploitation and subversion of plant defense: a lifestyle or just a phase, and implications in breeding resistance. Phytopathology 109: 332-346.

Lurie, S., E. Fallik, A. Handros, and R. Shapira, 1997 The possible involvement of peroxidase in resistance to Botrytis cinerea in heat treated tomato fruit. Physiol. Mol. Plant Pathol. 50: 141-149.

Margarido, G. R., A. P. Souza, and A. A. Garcia, 2007 OneMap: software for genetic mapping in outcrossing species. Hereditas 144: 78-79.

Meyer, D., E. Dimitriadou, K. Hornik, A. Weingessel, and F. Leisch, 2019 e1071: misc functions of the Department of Statistics, Probability Theory Group (Formerly: E1071), TU Wien. R package version 1.7-4. https://CRAN.R-project.org/package=e1071.

Mitcham, B., M. Cantwell, and A. Kader, 1996 Methods for determining quality of fresh commodities. Perishables Handling Newsletter 85: 1-5.

Moriya, Y., M. Itoh, S. Okuda, A. C. Yoshizawa, and M. Kanehisa, 2007 KAAS: an automatic genome annotation and pathway reconstruction server. Nucleic Acids Res. 35: W182-W185.

Pérez, P. and G. de Los Campos, 2014 Genome-wide regression and prediction with the BGLR statistical package. Genetics 198: 483-495.

Petrasch, S., S. J. Knapp, J. A. Van Kan, and B. Blanco-Ulate, 2019a Grey mould of strawberry, a devastating disease caused by the ubiquitous necrotrophic fungal pathogen Botrytis cinerea. Mol. Plant Pathol. 20: 877-892.

Petrasch, S., C. J. Silva, S. D. Mesquida-Pesci, K. Gallegos, C. van den Abeele, et al., 2019b Infection strategies deployed by Botrytis cinerea, Fusarium acuminatum, and Rhizopus stolonifer as a function of tomato fruit ripening stage. Front. Plant Sci. 10: 223.

Poland, J. and J. Rutkoski, 2016 Advances and challenges in genomic selection for disease resistance. Annu. Rev. Phytopathol. 54: 79-98.

Quidde, T., A. Osbourn, and P. Tudzynski, 1998 Detoxification of $\alpha$-tomatine by Botrytis cinerea. Physiol. Mol. Plant Pathol. 52: 151-165.

R Core Team, 2021 R: A Language and Environment for Statistical Computing. R Foundation for Statistical Computing, Vienna, Austria.

Rhainds, M., J. Kovach, and G. English-Loeb, 2002 Impact of strawberry cultivar and incidence of pests on yield and profitability of strawberries under conventional and organic management systems. Biol. Agric. Hortic. 19: 333-353.

Ries, S. M., 1995 Gray mold of strawberry. University of Illinois Integrated Pest Management Program Reports on Plant Diseases 704. http://ipm.illinois.edu/diseases/series700/rpd704/ .

Rowe, H. C. and D. J. Kliebenstein, 2008 Complex genetics control natural variation in Arabidopsis thaliana resistance to Botrytis 
cinerea. Genetics 180: 2237-2250.

Rueden, C. T., J. Schindelin, M. C. Hiner, B. E. DeZonia, A. E. Walter, et al., 2017 ImageJ2: ImageJ for the next generation of scientific image data. BMC Bioinformatics 18: 529.

Rutkoski, J. E., E. L. Heffner, and M. E. Sorrells, 2011 Genomic selection for durable stem rust resistance in wheat. Euphytica 179: 161-173.

Rutkoski, J. E., J. A. Poland, R. P. Singh, J. Huerta-Espino, S. Bhavani, et al., 2014 Genomic selection for quantitative adult plant stem rust resistance in wheat. Plant Genome 7: plantgenome2014.02.0006.

Saijo, Y., E. P.-i. Loo, and S. Yasuda, 2018 Pattern recognition receptors and signaling in plant-microbe interactions. Plant J. 93: 592-613.

Schiffthaler, B., C. Bernhardsson, P. K. Ingvarsson, and N. R. Street, 2017 BatchMap: A parallel implementation of the OneMap R package for fast computation of F1 linkage maps in outcrossing species. PloS one 12.

Schindelin, J., I. Arganda-Carreras, E. Frise, V. Kaynig, M. Longair, et al., 2012 Fiji: an open-source platform for biological-image analysis. Nat. Methods 9: 676-682.

Seijo, T. E., C. K. Chandler, J. C. Mertely, C. Moyer, and N. A. Peres, 2008 Resistance of strawberry cultivars and advanced selections to anthracnose and botrytis fruit rots. Proc. of the Florida State Hortic. Soc. 121: 246-248.

Silva, C. J., C. van den Abeele, I. Ortega-Salazar, V. Papin, J. A. Adaskaveg, et al., 2021 Host susceptibility factors render ripe tomato fruit vulnerable to fungal disease despite active immune responses. Journal of experimental botany 72: 2696-2709.

Silva, C. J., C. van den Abeele, I. O. Salazar, V. Papin, J. A. Adaskaveg, et al., 2020 Tomato fruit susceptibility to fungal disease can be uncoupled from ripening by suppressing susceptibility factors. bioRxiv 2020.06.03.132829.

Tomas-Grau, R. H., F. J. Requena-Serra, V. Hael-Conrad, M. G. Martínez-Zamora, M. F. Guerrero-Molina, et al., 2018 Soft mechanical stimulation induces a defense response against Botrytis cinerea in strawberry. Plant Cell Rep. 37: 239-250.

van Baarlen, P., L. Legendre, and J. A. van Kan, 2007 Plant defence compounds against Botrytis infection. In Botrytis: Biology, pathology and control, pp. 143-161, Springer.

van Kan, J. A., 2006 Licensed to kill: the lifestyle of a necrotrophic plant pathogen. Trends Plant Sci. 11: 247-253.

Van Os, H., P. Stam, R. G. Visser, and H. J. Van Eck, 2005 RECORD: a novel method for ordering loci on a genetic linkage map. Theor. Appl. Genet. 112: 30-40.

van Schie, C. C. and F. L. Takken, 2014 Susceptibility genes 101: how to be a good host. Annu. Rev. Phytopathol. 52: 551-581.

VanRaden, P., 2020 Symposium review: How to implement genomic selection. J. Dairy Sci. 103: 5291-5301.

Veloso, J. and J. A. van Kan, 2018 Many shades of grey in Botrytis host plant interactions. Trends Plant Sci. 23: 613-622.

Verma, S., J. D. Zurn, N. Salinas, M. M. Mathey, B. Denoyes, et al., 2017 Clarifying sub-genomic positions of qtls for flowering habit and fruit quality in us strawberry (fragaria $\times$ ananassa) breeding populations using pedigree-based qtl analysis. Horticulture research 4: 1-9.

Voss-Fels, K. P., M. Cooper, and B. J. Hayes, 2019 Accelerating crop genetic gains with genomic selection. Theor. Appl. Genet. 132: 669-686.

Williamson, B., B. Tudzynski, P. Tudzynski, and J. A. Van Kan, 2007 Botrytis cinerea: the cause of grey mould disease. Mol. Plant Pathol. 8: 561-580.
Zhang, W., J. A. Corwin, D. H. Copeland, J. Feusier, R. Eshbaugh, et al., 2019 Plant-necrotroph co-transcriptome networks illuminate a metabolic battlefield. eLife 8: e44279.

Zhang, Y., E. Butelli, R. De Stefano, H.-j. Schoonbeek, A. Magusin, et al., 2013 Anthocyanins double the shelf life of tomatoes by delaying overripening and reducing susceptibility to gray mold. Curr. Biol. 23: 1094-1100.

Zheng, Y., C. Jiao, H. Sun, H. G. Rosli, M. A. Pombo, et al., 2016 iTAK: a program for genome-wide prediction and classification of plant transcription factors, transcriptional regulators, and protein kinases. Mol. Plant 9: 1667-1670.

Zorrilla-Fontanesi, Y., A. Cabeza, P. Domínguez, J. J. Medina, V. Valpuesta, et al., 2011 Quantitative trait loci and underlying candidate genes controlling agronomical and fruit quality traits in octoploid strawberry (fragaria $\times$ ananassa). Theoretical and applied genetics 123: 755-778. 